\title{
IT'S NOT YOU, IT'S ME: COROLLARY DISCHARGE IN PRECEREBELLAR NUCLEI OF SLEEPING INFANT RATS
}

Didhiti Mukherjee ${ }^{1,5}$, Greta Sokoloff ${ }^{1,4,5}$, and Mark S. Blumberg ${ }^{1,2,3,4,5}$

${ }^{1}$ Department of Psychological and Brain Sciences, University of lowa, lowa City, lowa

${ }^{2}$ Interdisciplinary Graduate Program in Neuroscience, University of lowa, lowa City, lowa

${ }^{3}$ Department of Biology, University of lowa, lowa City, lowa

${ }^{4}$ lowa Neuroscience Institute, University of lowa, lowa City, lowa

${ }^{5}$ Delta Center, University of lowa, lowa City, lowa 


\section{ABSTRACT}

2 In week-old rats, somatosensory input arises predominantly from stimuli in the external

3 environment or from sensory feedback associated with myoclonic twitches during active

4 (REM) sleep. A previous study of neural activity in cerebellar cortex raised the possibility

5 that the brainstem motor structures that produce twitches also send copies of motor

6 commands (or corollary discharge, CD) to the cerebellum. Here, by recording from two

7 precerebellar nuclei-the inferior olive and lateral reticular nucleus-we demonstrate that

8 CD does indeed accompany the production of twitches. Within both structures, the CD

9 signal comprises a surprisingly sharp activity peak within $10 \mathrm{~ms}$ of twitch onset. In the

10 inferior olive, this sharp peak is attributable to the opening of slow potassium channels.

11 We conclude that a diversity of neural activity is conveyed to the developing cerebellum

12 preferentially during sleep-related twitching, enabling cerebellar processing of convergent

13 input from CD and reafferent signals. 


\section{INTRODUCTION}

2 The sensorimotor systems of diverse vertebrate and invertebrate species distinguish

3 signals arising from self-generated movements (i.e., reafference) from those arising from

4 other-generated movements (i.e., exafference; Cullen, 2004). To make this distinction,

5 motor structures generate copies of motor commands, referred to as corollary discharge

6 (CD; Crapse and Sommer, 2008; Poulet and Hedwig, 2007). CD is conveyed to non-motor

7 structures to inform them of the imminent arrival of reafference arising from self-generated

8 movements (Crapse and Sommer, 2008). By comparing the two signals, animals are able

9 to distinguish between self-produced and other-produced movements.

10 Self-produced movements are not restricted to periods of wakefulness, especially

11 during development. Infants produce brief, discrete, jerky movements of skeletal muscles

12 during active sleep (AS or REM sleep), which is the predominant behavioral state during

early infancy (Jouvet-Mounier et al., 1970; Roffwarg et al., 1966). These spontaneous

14 movements are known as myoclonic twitches, and are among the most conspicuous

15 behaviors during development in a diversity of species (Blumberg et al., 2013;

16 Gramsbergen et al., 1970; Jouvet-Mounier et al., 1970; Roffwarg et al., 1966).

17 It was recently reported in week-old rats that the external cuneate nucleus (ECN), a

medullary nucleus that receives proprioceptive input from the forelimbs, actively inhibits

reafference arising from wake-related limb movements but not those arising from twitches

20 (Tiriac and Blumberg, 2016). This state-dependent gating by the ECN suggested the

21 selective engagement of a CD mechanism during wake movements and its suspension

22 during twitching. It remained unclear, however, whether the suspension of the gating

23 mechanism reflected the absence of a twitch-related CD signal or the inhibition of CD's 
1 effects within the ECN. Determining whether twitches are accompanied by CD has

2 implications for their functional role in the self-organization of the sensorimotor system.

3 There is some suggestive evidence that twitches are accompanied by CD. In week-old

4 rats, twitches trigger both complex spikes (arising from climbing fibers) and simple spikes

5 (arising from mossy fibers) in cerebellar Purkinje cells (Sokoloff et al., 2015a). These

6 neural events were detected at very short latencies that appear too short for reafferent

7 signals arising from the periphery (Puro and Woodward, 1977a; Puro and Woodward,

8 1977b). Accordingly, it is possible that the motor structures that produce twitches also

9 convey CD to the cerebellum, as occurs with waking movements in adults (Azim and

10 Alstermark, 2015; Azim et al., 2014).

11 If twitch-related CD reaches cerebellar cortex, it must be conveyed through the precerebellar nuclei. The inferior olive $(\mathrm{IO})$ is a good candidate structure for such a CD signal. First, it is the sole source of climbing fibers to cerebellar cortex and is therefore responsible for the triggering of complex spikes (Ruigrok et al., 2014). Second, midbrain motor structures, including Nucleus of Darkschewitsch, project directly to the IO (De Zeeuw et al., 1998). Finally, the 10 fires precisely at the onset of self-generated movements in waking adults (Keating and Thach, 1995; Welsh et al., 1995).

With respect to mossy fibers, there are a few major candidate structures to consider (Ruigrok et al., 2014). First, the pontine nucleus is an unlikely source of CD in week-old rats because it receives descending input from motor cortex (Lee and Mihailoff, 1990),

21 which does not contribute to the production of twitches (Blumberg, 2010; Kreider and 22 Blumberg, 2000). Second, the ECN can also be ruled out as a source of CD because it 23 has been demonstrated to process twitch-related reafference exclusively (Tiriac and 
1 Blumberg, 2016). Finally, the LRN is a viable candidate because it receives both sensory

2 input from the limbs and motor input from midbrain structures, including the red nucleus

3 (Alstermark and Ekerot, 2013; Pivetta et al., 2014). Moreover, the LRN has been

4 implicated in processing $C D$ associated with self-generated movements in adults

5 (Alstermark and Ekerot, 2015; Arshavsky et al., 1978).

6 Accordingly, we recorded neural activity in the IO and LRN in postnatal day (P) 7-9

7 (hereafter P8) rats. In the majority of IO units and a subset of LRN units, twitch-related

8 activity was remarkably precise, exhibiting a sharp activity peak within $\pm 10 \mathrm{~ms}$ of a twitch.

9 This sharp peak, centered so tightly around twitch onset, is most parsimoniously explained

10 as CD. Next, we identified adjacent, non-overlapping midbrain motor areas that are

11 involved in the production of twitches and that are likely sources of CD to the IO and LRN.

12 Finally, we demonstrate that calcium-activated slow potassium (SK) channels in the $\mathrm{IO}$ are

13 responsible for sharpening the broad CD signal arriving from motor structures.

14 Altogether, the twitch-related activity peaks in the IO and LRN reported here satisfy the 15 major criteria put forward for identifying CD (Poulet and Hedwig, 2007; Sommer and Wurtz, 16 2008). To our knowledge, these findings provide the first direct neurophysiological 17 evidence of a CD signal in an infant mammal.

\section{IO activity predominates during active sleep}

21 We recorded IO activity in unanesthetized head-fixed pups as they cycled spontaneously 22 between sleep and wake with their limbs dangling freely ( $n=20$ pups, 37 units, 1-4 23 units/pup). Electromyography (EMG) and behavioral scoring were used to identify 
1 behavioral state and detect sleep and wake movements (Blumberg et al., 2015; Figure

$21 \mathrm{~A})$.

3 Electrode placement within the $\mathrm{IO}$ was confirmed histologically (Figure 1B). Recording

4 sites were located within the dorsal accessory olive (DAO; n=19 units across 12 pups) or

5 the medial accessory olive (MAO) and the principal olive (PO; $n=18$ units across 8 pups;

6 Figure 1C). Overall, unit activity was phasic and largely restricted to periods of AS; also,

7 activity appeared to be suppressed immediately after the onset of active wake (AW).

8 Sparse activity was observed during behavioral quiescence (BQ), which is a period of low

9 muscle tone interposed between AW and AS (Figure 1D). The majority (24/35 units, 69\%)

10 of IO units were AS-on (Figure 1E) and the mean firing rate of the AS-on units $(2.9 \pm 0.4$

$11 \mathrm{~Hz}$ ) was approximately three times higher during AS than for the other two states

$12(p<0.0005 ;$ Figure 1F). Two IO units were excluded from state analysis due to movement 13 artifact during AW.

\section{IO neurons exhibit sharp activity peaks at twitch onset}

The phasic 10 activity clustered around periods of myoclonic twitching; therefore, we examined the temporal relationship between twitches and unit activity by creating perievent histograms (5-ms bins, 1-s window) with unit activity triggered on twitch onset.

Previous studies have revealed two distinct patterns of twitch-triggered perievent histograms in sensorimotor structures (Figure 2A). First, in a motor structure like the RN

21 (green trace in Figure 2A), unit activity increases 20-40 ms before the onset of a twitch 22 (Del Rio-Bermudez et al., 2015). Second, in a sensory structure like the ECN (blue trace 23 in Figure 2A), unit activity increases at least 10-50 ms after the onset of a twitch (Tiriac 
1 and Blumberg, 2016). In the IO, however, we found that the majority of units (23/37 units,

$262 \%$ ) were active within \pm 10 ms of the onset of a twitch (Figure $2 \mathrm{~B}, \mathrm{C}$ ). These IO activity

3 profiles were very sharp and thus strikingly different from those observed in any of the

4 motor and sensory structures from which we have previously recorded (e.g., see Figure

5 2D). Also, the $\mathrm{IO}$ units that exhibited this profile were responsive primarily to nuchal and/or

6 forelimb twitches and rarely to hindlimb twitches (Figure 2-figure supplement 1A-D).

7 Finally, the characteristics of the neural responses recorded in the 10 did not appear to

8 differ across anatomical subdivisions.

$9 \quad$ There are three possible explanations for these sharply peaked activation patterns

observed in 10 units: (a) the $1 \mathrm{O}$ is part of the motor pathway, (b) the $\mathrm{IO}$ receives reafference

11 from twitches, and (c) the $\mathrm{IO}$ receives $\mathrm{CD}$ from a motor structure that produces twitches.

With respect to $(a)$, the 10 , despite being implicated in the precise timing of motor

behaviors (De Zeeuw et al., 1998), is not directly involved in the generation of movements

14 (Horn et al., 2004; Lang et al., 2017). Although it receives afferent projections from motor areas (Saint-Cyr, 1983; Saint-Cyr and Courville, 1981), there are no efferent projections

16 from the $\mathrm{IO}$ to spinal motor neurons. In fact, the sole efferent projection from the $1 \mathrm{O}$

17 comprises the climbing fibers that innervate cerebellar Purkinje cells (Ruigrok et al., 2014).

18 Consequently, in adults, stimulation of the $\mathrm{IO}$ does not evoke or modulate movements 19 (Gellman et al., 1985).

With respect to (b), although the 10 can receive short-latency reafferent signals 21 (Gellman et al., 1983; Sedgwick and Williams, 1967), it is unlikely that reafference can account for the short-latency peaks observed here. Consider that for the structures in 23 which we have seen clear evidence of twitch-related reafference (e.g., ECN), we have also 
1 seen clear evidence of exafferent responses (Tiriac et al., 2014; Tiriac and Blumberg,

2 2016). In contrast, of the 10 units that exhibited sharp peaks with a latency of $\pm 10 \mathrm{~ms}$,

3 none responded to exafferent stimulation. Moreover, of the $7 \mathrm{IO}$ units that exhibited twitch-

4 triggered responses at latencies consistent with reafferent processing (i.e., > 10 ms; Figure

5 2-figure supplement 1E), 3 responded to exafferent stimulation. Thus, the signature

6 feature of the majority of 10 activity-the sharp peak centered on twitch onset-is

7 consistent with the notion that the $I O$ receives $C D$ associated with the production of a

8 twitch.

9

10 LRN neurons exhibit two kinds of twitch-related activity

11 Based on research in adults (Alstermark and Ekerot, 2015; Arshavsky et al., 1978), we predicted that the LRN, like the IO, would exhibit CD-related activity. Moreover, because

13 the LRN also receives sensory inputs from the limbs (Figure 3A), we expected to see 14 evidence of reafference in that structure. To test these two possibilities, we next recorded spontaneous LRN activity in P8 rats across sleep and wake.

We confirmed electrode placements in the LRN ( $n=27$ units across 9 pups, 1-6

17 units/pup; Figure 3B). Similar to the IO, the unit activity in the LRN was phasic and 18 restricted to periods of AS, particularly around twitches. LRN activity was sparse during $19 \mathrm{BQ}$ and was suppressed after AW onset (Figure 3C). The majority (16/27 units, 59\%) of

20 LRN units were AS-on (Figure 3D) and the mean firing rate of the AS-on units (1.4 \pm 0.2 $21 \mathrm{~Hz}$ ) was approximately three times higher during AS than during any of the other two states $22 \quad(p=<0.0005$; Figure 3E). 
Next, we assessed the temporal relationship between unit activity and twitches by creating perievent histograms (5-ms bins, 1-s window). Regardless of state dependency,

3 the majority of LRN units (24/27 units, $89 \%)$ showed significant increases in firing rate in

4 response to a twitch (Figure 3F). As predicted, we observed two different neural

5 populations the exhibited distinct patterns of twitch-triggered activity. First, we found a

6 subpopulation of LRN units (12/27 units, 44\%) that, like the majority of IO units, exhibited

7 a sharp peak within \pm 10 ms of a twitch (Figure $3 G$, left); also, none of these LRN units

8 responded to exafferent stimulation of the limbs (data not shown).

Second, the remaining LRN units (12/27 units, 44\%) exhibited broader twitch-related activity profiles consisting of a peak in activity around twitch onset $( \pm 10 \mathrm{~ms})$ and/or a peak

11 with a latency of $>10 \mathrm{~ms}$ (Figure $3 \mathrm{G}$, right). The latter peak is what is expected from a short-latency reafferent responses (Tiriac and Blumberg, 2016; Tiriac et al., 2014). Indeed,

6 of these 12 units also responded to exafferent stimulation of either the forelimb or hindlimb with an average latency of $40 \mathrm{~ms}$ (Figure $3 \mathrm{H})$.

\section{Non-overlapping regions in the mesodiencephalic junction (MDJ)}

18 The MDJ includes diverse structures, like the RN, that innervate spinal motor neurons (De

Zeeuw et al., 1998; Saint-Cyr and Courville, 1981; Lakke, 1997; Onodera and Hicks, 2009;

Zuk et al., 1983) and are therefore directly involved in the generation of movements

21 (Fukushima, 1991; Morris et al., 2015; Onodera and Hicks, 1996; Williams et al., 2014).

22 To determine whether MDJ neurons also project to the IO and LRN at P8, we performed retrograde tracing from each structure. 
Wheat germ agglutinin (WGA) conjugated with Alexa Fluor 488 or 555 was microinjected into the $\mathrm{IO}$ or LRN and retrogradely labelled cell bodies were imaged using

3 a fluorescent microscope. Retrograde tracing from the $\mathrm{IO}(\mathrm{n}=5$; Figure $4 \mathrm{~A})$ revealed robust

4 labeling of cell bodies in the mesodiencephalic junction (MDJ), including the rostral

5 interstitial nucleus of Cajal (RI), nucleus of Darkschewitsch (Dk), accessory oculomotor

6 nuclei (MA3), and nearby diffuse areas in the MDJ. Little or no labelling was observed in

7 the RN. In contrast, retrograde tracing from the LRN ( $n=4$; Figure 4B) revealed robust

8 labeling in the contralateral RN but not elsewhere in the MDJ. Finally, when the two

9 different tracers were injected separately into the IO and LRN of the same pup $(\mathrm{n}=2$; Figure

$104 \mathrm{C}$ ), we found that the LRN-projecting cell bodies were located within the RN and the IO-

11 projecting cell bodies were located outside the RN. These findings are consistent with

12 those in adult rats (Ruigrok et al., 2014) and suggest that the major afferent connections

13 to the IO and LRN arise from non-overlapping structures in the MDJ at this age.

MDJ stimulation causes limb movements and c-Fos activation in the IO

\section{and LRN}

To assess functional connectivity between the MDJ and the IO or LRN, we electrically stimulated the RN $(n=4)$ and other MDJ nuclei $(n=4)$ while monitoring forelimb and hindlimb movements in urethanized (1.5mg/g, IP) P8 rats (Figure 4-figure supplement 1A). Subsequently, we performed immunohistochemistry to determine the expression of the c21 Fos protein, a marker of neural activity (Chung, 2015), in the IO and LRN.

Stimulation of several non-RN MDJ nuclei evoked non-specific movements of the 
1 primarily in the ipsilateral IO (Figure 4-figure supplement 1B). In contrast, stimulation of

2 the RN produced only discrete contralateral forelimb movements (Figure 4-figure

3 supplement $1 \mathrm{~A}$ ) and resulted in c-Fos expression in the contralateral LRN but not the IO

4 (Figure 4-figure supplement 1C). These results indicate that MDJ nuclei are functionally

5 connected to the IO and LRN at these ages.

6 It is possible that the c-Fos activation in the IO and LRN was due to sensory feedback

7 arising from the stimulated movements. However, we observed little or no c-Fos

8 expression in sensory areas like the cuneate nucleus and ECN (data not shown).

MDJ neurons adjacent to the RN are active before and after the

\section{production of twitches}

It has been established that the RN is involved in the production of twitches (Del Rio-

Bermudez et al., 2015) and, as shown here, in the conveyance of CD to the LRN. Similarly,

if MDJ neurons outside of the RN convey twitch-related CD to the IO, we would also expect

these neurons to be involved in the production of twitches (Figure 5A). Therefore, we

characterized the spontaneous activity of non-RN MDJ neurons in P8 rats during sleep

and wake. We aimed to record from MDJ neurons located in regions implicated earlier as

projecting to the $\mathrm{IO}$ and, upon stimulation, producing limb movements (see Figure 4 and

Figure 4-figure supplement 1).

Electrode placements in the MDJ immediately medial to the RN were confirmed $(n=7$

pups, 17 units, 1-5 units/pup; Figure 5B). The spontaneous activity of neurons in this

22 region appeared mostly around twitches and wake movements (Figure 5C). When twitch-

23 triggered perievent histograms (10-ms bins, 1-s window) were created, we found that the 
1 majority of the recorded units (15/17 units, $88 \%)$ showed significant twitch-dependent

2 activity (Figure 5D).

3 The temporal relationship between neural activity and twitches revealed two primary

4 subpopulations of units (Figure 5D): There were units that significantly increased their

5 firing rates before the onset of a twitch (twitch-preceding units) and units that significantly

6 increased their firing rates after the onset of a twitch (twitch-following units). The twitch-

7 preceding units (10/17 units across 5 pups, $59 \%)$ showed an increase in firing rate $10-70$

8 ms before a twitch (Figure 5E, left). Interestingly, the majority (7/10 units, 70\%) of these

9 twitch-preceding units also exhibited an increase in firing rate 10-50 ms after a twitch,

10 indicative of reafference. The twitch-following units (5/17 units across 3 pups, 29\%)

11 showed an increase in firing rate 20-40 ms after a twitch (Figure 5E, right), suggesting they

12 only receive reafference from a twitch. In terms of pattern and latency of twitch-triggered

13 activity, these neurons behave similarly to those described previously in the RN (Figure

14 5F; Del Rio-Bermudez et al., 2015).

15 The twitch-preceding MDJ neurons also significantly increased their activity before the 16 onset of wake movements (Figure 5-figure supplement 1A), consistent with that observed

17 in the RN (Del Rio-Bermudez et al., 2015). Similarly, the IO units that exhibited twitch-

18 related $C D$ also significantly increased their firing rate at the onset of wake movements

19 (Figure 5-figure supplement 1B). A similar pattern was observed in the LRN (data not 20 shown). Therefore, the IO and LRN receive CD from the MDJ associated with both types

21 of movements, although wake-related activity is less abundant and pronounced than 22 twitch-related activity. 


\section{Calcium-activated slow-potassium (SK) channels contribute to the}

\section{2 sharp peak in IO activity}

3 Having identified motor structures that send $C D$ to the IO and LRN, we next sought to

4 determine how a motor command with a broad twitch-preceding peak (see Figure 5F) is

5 transformed into a sharp, precise peak around a twitch (see Figure 2B). We focused on

6 the $\mathrm{IO}$ to address this question because of the reliably high percentage of units that exhibit

7 twitch-related CD.

8 In the adult IO, SK channels prevent temporal summation of excitatory presynaptic

9 inputs (Garden et al., 2017). SK channels are also expressed early in development 10 (Gymnopoulos et al., 2014). Because afferent projections from the MDJ to the IO are 11 excitatory, we hypothesized that twitch-related CD conveyed to the IO is accompanied by

12 the opening of SK channels, thereby truncating IO activity and resulting in the observed 13 sharp twitch-related peaks. To test this hypothesis, we blocked SK channels using apamin, 14 an SK channel antagonist (Benington et al., 1995); apamin has been used in adult rats to block SK channels in the IO (Lang et al., 1997).

P8 rats were prepared for neurophysiological recording as described earlier. After the pup was cycling between sleep and wake, apamin ( $1 \mu \mathrm{M}$; dissolved in saline) or salinemixed with $4 \%$ fluorogold to later identify the extent of diffusion-was microinjected at a volume of $100 \mathrm{nl}$ into the IO. Fifteen min after the injection (the half-life of apamin is $\sim 2 \mathrm{~h}$;

20 Gui et al., 2012), the microsyringe was withdrawn and was replaced with a recording 21 electrode. Neural and EMG activity and sleep-wake behavior were then recorded for 30 $22 \min$ (Figure 6A). 
We confirmed drug or vehicle diffusion and recording sites within the 10 ( $n=18$ units across 10 pups in the saline group; $n=21$ units across 8 pups in the apamin group; $1-3$

units/pup; Figure 6B). There was no difference in the amount of time spent in $A S(p=0.78)$

4 or in the number of twitches produced per unit time of AS between the apamin and saline

5 groups (ps>0.15 for nuchal, contralateral and ipsilateral forelimb twitches; Figure 6-figure

6 supplement 1 A, B). As observed in the previous 10 recordings, neural activity in both

7 groups was phasic and restricted to periods of AS (Figure 6-figure supplement 1C).

8 There was no significant difference in the overall firing rate during AS between groups

$9 \quad(p=0.59 ;$ Figure 6-figure supplement 1D).

Perievent histograms (10-ms bins, 1-s window) were created for each individual unit in

11 both groups (Figure 6C). As predicted, whereas twitch-triggered activity in the saline group

exhibited the expected sharp peak around twitches, the activity in the apamin group was

broader during the period after twitch onset.

The number of units exhibiting significant twitch-related activity did not differ between

the two groups $\left(n=13 / 18\right.$ in saline and $n=11 / 21$ in apamin groups; $X^{2}(1, N=39)=1.6, p=0.2$;

Figure 6D). In contrast, the number of units exhibiting sharp peaks within $\pm 10 \mathrm{~ms}$ of a twitch was significantly lower in the apamin group (5/21 units, $24 \%)$ than in the saline group $\left(11 / 18\right.$ units, $61 \% ; X^{2}(1, N=39)=5.6, p=0.02 ;$ Figure $\left.6 D\right)$. To illustrate the effect of apamin on twitch-related activity, we pooled the data for the significant units to create perievent histograms of $1 \mathrm{O}$ activity. As shown in Figure 6E, the activity in the apamin group, unlike

21 that in saline group, persisted beyond the 10-ms window after a twitch. To quantify the difference, we calculated the area under the curve for each unit during two time windows:

$23 \pm 10 \mathrm{~ms}$ around a twitch and $20-200 \mathrm{~ms}$ after a twitch (Figure 6F). As expected, we found 
1 no significant difference between the two groups in the \pm 10 -ms window $(U=56.5, Z=-0.87$,

$2 \mathrm{p}=0.4$ ), but did find a significant difference for the 20-200-ms window, with the apamin

3 group being significantly larger $(U=30.5, Z=-2.2, p=0.03)$. In fact, the pattern of twitch-

4 triggered neural activity in the apamin group was similar to that recorded in the MDJ

5 (Figure 6G). Based on these results, we conclude that SK channels are involved in

6 sharpening the CD signal arriving from the MDJ.

8 Discussion

9 Several criteria have been proposed for identifying CD signals (Poulet and Hedwig, 2007;

10 Sommer and Wurtz, 2008). First, a CD should originate in a structure that is demonstrably

11 involved in the production of movement; as shown here, the twitch-related activity in the

12 IO and LRN originates from several independent motor structures in the MDJ that are

13 involved in the production of twitches and wake movements (Del Rio-Bermudez et al.,

14 2015). Second, areas receiving CD should themselves play no direct role in the production

15 of movement; this is clearly true of the IO and LRN (Gellman et al., 1985; Ruigrok et al.,

16 2014). Third, neurons receiving CD should increase their activity at the onset of a

17 movement; as shown here, the activity of IO and LRN neurons occurs precisely at the

18 onset of twitches, exhibiting a temporal profile that clearly distinguishes it from twitch-

19 preceding activity in the MDJ nuclei and twitch-following activity in the ECN. Thus, the

20 twitch-related activity in the IO and LRN satisfies the key criteria of CD. Below we discuss

21 the implications of this finding and its significance for sensorimotor development. 
$1 \quad$ Neural pathways conveying CD have been delineated in a diverse array of species (Dale

2 and Cullen, 2017; Davis et al., 1973; Fee et al., 1997; Schneider et al., 2014; Sommer and

3 Wurtz, 2002; Yang et al., 2008). Neural recordings of the CD signal itself, however, have

4 mostly been performed in non-mammalian species, including crickets, sea slugs, crayfish,

5 tadpoles, and electric fish (Evans et al., 2003; Kirk and Wine, 1984; Li et al., 2004; Poulet

6 and Hedwig, 2006; Requarth and Sawtell, 2014). The relatively small and simple nervous

7 systems of these species have allowed for the isolation of neurons that carry or receive

8 CD signals and identify their relationship to behavior. In contrast, CD signals have thus far

9 only been recorded in the mediodorsal thalamus of non-human primates during eye

10 movements (Sommer and Wurtz, 2004) and the auditory cortex of mice (Schneider et al.,

$112014)$

The current findings provide the first direct neurophysiological evidence of CD in a

developing mammal. Moreover, this is the first direct evidence of CD in the IO and LRN,

consistent with what has been proposed for these two structures (Alstermark and Ekerot,

2013; Arshavsky et al., 1978; De Zeeuw et al., 1998; Devor, 2002). Also, with this

16 discovery of a unique neural CD signature-comprising a short-latency onset and sharp

17 activity peak — we have a clear template to guide future neurophysiological investigations

18 of CD signals in other species and neural systems across the lifespan.

\section{A neural mechanism for sharpening the CD signal}

21 As mentioned above, one of the signature features of the twitch-related CD signal is the

22 sharp peak. This is surprising because, as shown here and in a previous study (Del Rio-

23 Bermudez et al., 2015), twitch-related motor activity in MDJ neurons exhibits broad peaks 
1 (see Figure 5F). How does a broad presynaptic signal in the MDJ get converted into a

2 sharp postsynaptic response in the IO and LRN (see Figures 2C, 3G)? To answer this

3 question, we focused on the 10 because, compared with the LRN, a much higher

4 proportion of its neurons exhibited sharp peaks.

$5 \quad$ There are a few possible candidate mechanisms. For example, in cortical pyramidal

6 neurons, interactions between excitatory and inhibitory inputs can sharpen a neuron's

7 activity profile (Kremkow et al., 2010). A similar mechanism is unlikely to operate in the IO

8 for several reasons. First, inhibitory interneurons are sparse in that structure $(<0.1 \%$;

9 Nelson and Mugnaini, 1988). Second, although the 10 receives its predominant inhibitory

10 input from the deep cerebellar nuclei (DCN; de Zeeuw et al., 1988), DCN activity occurs

$11 \sim 40 \mathrm{~ms}$ after a twitch (Del Rio-Bermudez et al., 2016). Moreover, in pilot experiments, we

12 found that pharmacological inactivation of the DCN had no effect on $\mathrm{IO}$ activity at P8,

13 consistent with a previously published report (Nicholson and Freeman, 2003).

14 Consequently, we hypothesized that inhibition in the IO is mediated by SK channels.

15 In the 10 of adult rats, these channels prevent summation of excitatory inputs (Garden et

16 al., 2017). Here, using pharmacological inactivation, we demonstrate that SK channels are

17 responsible for sharpening the olivary CD signal. A similar mechanism could be functional

18 in the LRN as SK channels are also expressed in that structure in adult rats (Xu et al., 19 2013).

\section{Differential actions of CD signals at precerebellar nuclei}

22 In a previous study (Tiriac and Blumberg, 2016), it was demonstrated that wake-related

23 reafference is blocked within the ECN, an effect that we attributed to modulation by a 
1 wake-related CD signal. That CD-mediated blockade was lifted during twitching, thereby

2 allowing twitch-related reafference to be conveyed to downstream motor structures,

3 including the cerebellum. In contrast, focusing here on the IO and LRN, we found that the

4 twitch-related CD signals themselves_not reafference-are conveyed to the cerebellum

5 (Figure 7A). Therefore, within this broader context, we see that CD accompanies sleep

6 and wake behavior in infants, but its effects are not monolithic: It can modulate the action

7 of a comparator to gate reafference (as in the ECN) or be transmitted sequentially to

8 multiple downstream structures (as in the IO or LRN $\rightarrow$ cerebellum). Such diverse effects

9 of CD have been described elsewhere (Crapse and Sommer, 2008).

Although the three precerebellar nuclei-IO, LRN, and ECN-process CD and

11 reafference differently, the common denominator of all this activity is the inundation of the

12 developing cerebellum with twitch-related information (Figure 7A). In contrast, wake-

13 related activity has been shown to be blocked at the level of the ECN (Tiriac and Blumberg,

14 2016), thereby accounting in part for the relatively low levels of activity in the cerebellum

15 at this age (Sokoloff et al., 2015a; Sokoloff et al., 2015b). In addition, we found here that

16 the IO and LRN are also considerably less active during periods of wake. Although wake

17 movements were occasionally accompanied by bursts of IO and LRN activity, this activity

18 was much less pronounced than that observed at the onset of twitching. These weaker

19 activation patterns could simply be due to the fact that wake movements, compared to

20 twitches, are fewer in number at this age. For example, for a typical limb muscle in a P8

21 rat over a 30-min period, we find that there are $\sim 300$ twitches but only $~ 30$ wake

22 movements. Consequently, when compared with twitches, there are many fewer 
1 opportunities for wake movements to activate downstream cerebellar structures (Figure

2 7B).

\section{Functional implications of CD and reafference}

5 The developing sensorimotor system receives substantial sensory input from self-

6 generated twitches and from external stimulation arising from the mother and littermates.

$7 \quad$ It has been suggested that the infant brain does not distinguish between these two sources

8 of input and that twitch-related reafference serves merely as a "proxy" for exafferent

9 stimulation (Akhmetshina et al., 2016; McVea et al., 2016). This suggestion rests in part

on the observation that both forms of stimulation, despite their very different origins, trigger

11 similar patterns of cortical activity (Akhmetshina et al., 2016; Tiriac et al., 2012; Yang et

al., 2013). Thus, with our finding that CD accompanies the production of twitches, it is now

clear that there exists a mechanism with which the infant brain can distinguish self-

generated from other-generated movements; the ability to make this distinction is thought

to rely in part on the cerebellum (Blakemore et al., 2000; Wolpert et al., 1998).

There are a number of ways in which twitch-related CD could contribute to cerebellar development and function. For example, in the adult cerebellum, CD and reafference are known to converge via climbing and mossy fibers (Blakemore et al., 2001; Huang et al., 2013; van Kan et al., 1993; Wolpert et al., 1998). In this way, it is thought that the cerebellum instantiates a forward model that receives sensory predictions and computes

21 prediction errors (by comparing CD with reafference) in order to facilitate motor learning

22 (Blakemore et al., 2000; Brooks et al., 2015; Requarth and Sawtell, 2014; Wolpert et al., 23 1998). 
Twitches could contribute to the process by which forward models are instantiated and updated, especially in the context of a rapidly growing body. To appreciate this possibility,

consider this description of cerebellar function: "After much trial and error during infancy

and throughout life, the cerebellum learns to associate actual movements with intended

movements. Many of our motor memories are movements that we have repeated millions

6 or billions of times..." (p. 538, Mason, 2011). In that context, the millions of twitches

7 produced in early infancy could be a critical source of repeated convergent input to the

8 developing cerebellum. This convergence, illustrated in Figure 7, would provide the

9 developing cerebellum with abundant opportunities to align prediction and feedback

10 signals in a topographically organized fashion.

11 Cerebellar circuitry undergoes substantial development over the first three postnatal

weeks in rats (Altman, 1972a; b; c; Shimono et al., 1976; Wang and Zoghbi, 2001). Many

of these developmental processes depend heavily on neural activity, including climbing

fiber synapse elimination and translocation at Purkinje cells (Andjus et al., 2003; Kakizawa

et al., 2000; Kano and Hashimoto, 2012; Watanabe and Kano, 2011). With respect to

synapse elimination, beginning around P8, the initial multiple innervation of Purkinje cells

by climbing fibers begins to shift toward singly innervated cells in the second postnatal week as one climbing fiber is selectively strengthened over others. Importantly, spike timing-dependent plasticity (STDP) has been implicated in this process (Kawamura et al., 2013); STDP depends on the repetitive and sequential firing of pre- and post-synaptic cells

21 within a short and precise time window (Feldman, 2012; Kawamura et al., 2013; Sgritta et al., 2017). The present findings in precerebellar nuclei, in which twitch-related CD reliably 
1 role in cerebellar development via STDP. Moreover, recording from Purkinje cells at P8,

2 we previously found that complex and simple spikes were highly likely to occur within 0-

350 ms after twitches (Sokoloff et al., 2015a).

\section{Implications for neurodevelopmental disorders}

6 Disruption of cerebellar function during sensitive periods of development can have

7 negative cascading effects on cerebello-cortical communication and ultimately on

8 associated sensorimotor and cognitive processes, as observed in autism spectrum

9 disorder (Diamond, 2000; Wang et al., 2014). There are many potential causes of early

cerebellar dysfunction, including prenatal and postnatal exposure to environmental

11 stressors (Wang et al., 2014). One such stressor could be sleep deprivation or restriction,

especially during early infancy when sleep is the predominant state (Jouvet-Mounier et al., 1970; Roffwarg et al., 1966). As demonstrated here and in previous studies (Sokoloff et

in early development. Therefore, perturbation of sleep could deprive the cerebellum and other structures of critical sensorimotor activity during sensitive periods of development.

Accumulating evidence also suggests that CD-related processing is dysfunctional in patients with schizophrenia. Specifically, failure to disambiguate "self-generated" from "other-generated" sensory input may underlie hallucinations and delusions of control (Feinberg and Guazzelli, 1999; Ford et al., 2008). If twitches help to instruct the developing

21 brain to distinguish self from other, disruption of sleep and sleep-related sensorimotor 


\section{Conclusion}

2 It has been argued that the discreteness of twitches makes them ideally suited to provide

3 high-fidelity sensory information at ages when activity-dependent development is so

4 important for the developing nervous system (Blumberg et al., 2013; Tiriac et al., 2015).

5 The present results go further to suggest that the convergence of twitch-related CD and

6 reafference associated with millions of twitches over the early developmental period

7 provides ample opportunity for assimilating growing limbs into the infant's emerging body

8 schema (Blumberg and Dooley, 2017).

\section{MATERIALS AND METHODS}

11 All experiments were carried out in accordance with the National Institutes of Health Guide

12 for the Care and Use of Laboratory animals (NIH Publication No. 80-23). Experiments

13 were also approved by the Institutional Animal Care and Use Committee (IACUC) of the

14 University of lowa.

\section{Subjects}

17 Male and female Sprague-Dawley Norway rats (Rattus norvegicus) at postnatal day (P) 7-

189 (hereafter P8; n=68) from 60 litters were used for the study. All litters were culled to eight

19 pups by P3. Mothers and litters were housed and raised in standard laboratory cages (48

$20 \times 20 \times 26 \mathrm{~cm}$ ). Food and water were available ad libitum. The animals were maintained on

21 a 12-h light-dark cycle with lights on at $0700 \mathrm{~h}$. Littermates were never assigned to the 22 same experimental group. 


\section{Surgery}

3 A pup with a visible milk band was removed from the home cage. Under isoflurane (3-5\%)

4 anesthesia, bipolar hook electrodes (50 $\mu \mathrm{m}$ diameter, California Fine Wire, Grover Beach,

5 CA) were inserted into the nuchal, forelimb, and hindlimb muscles for electromyography

6 (EMG) and secured with collodion. A stainless steel ground wire was secured transdermally

7 on the back. A custom-built head-fix device was then secured to the exposed skull with

8 cyanoacrylate adhesive (Blumberg et al., 2015). The local anesthetic, Bupivicaine $(0.25 \%)$

9 was applied topically to the site of incision and some subjects were also injected

10 subcutaneously with the analgesic agent carprofen $(0.005 \mathrm{mg} / \mathrm{g})$. The pup was lightly

11 wrapped in gauze and allowed to recover in a humidified, temperature-controlled $\left(35^{\circ} \mathrm{C}\right)$

12 incubator for at least one hour. After recovery, the pup was briefly ( $<15 \mathrm{~min})$ re-anesthetized

13 with isoflurane (2-3\%) and secured in a stereotaxic apparatus. A hole was drilled in the

14 skull for insertion of the recording electrode into the inferior olive (IO; coordinates: $A P=3.4-$

$153.6 \mathrm{~mm}$ caudal to lambda; $M L=0-1.2 \mathrm{~mm}$ ), the lateral reticular nucleus ( $\mathrm{LRN}$; coordinates:

$16 \mathrm{AP}=3.5-3.7 \mathrm{~mm}$ caudal to lambda; $\mathrm{ML}=1.5-1.8 \mathrm{~mm}$ ), or midbrain nuclei near the red nucleus

17 (RN) within the mesodiencephalic junction (MDJ; coordinates: AP=4.7-4.9 mm caudal to

18 bregma; $M L=0.2-0.5 \mathrm{~mm}$ ). Two additional holes were drilled over the frontal or parietal

19 cortices for subsequent insertion of the ground wire and a thermocouple (Omega

20 Engineering, Stamford, CT) to measure brain temperature. In 15 pups in which no

21 neurophysiological recordings were performed, only one additional hole was drilled for

22 insertion of the thermocouple. After surgery, the pup was transferred to the recording

23 chamber. 


\section{Electrophysiological recordings}

3 The head-fix device was secured to the stereotaxic apparatus housed within the recording

4 chamber and the pup was positioned with its body prone on a narrow platform with limbs

5 dangling freely on both sides (Blumberg et al., 2015). Care was taken to regulate air

6 temperature and humidity such that the pup's brain temperature was maintained at 36-37

${ }^{\circ} \mathrm{C}$. Adequate time $(1-2 \mathrm{~h})$ was allowed for the pup to acclimate to the recording

8 environment and testing began only when it started cycling normally between sleep and

9 wake. Pups rarely exhibited abnormal behavior or any signs of discomfort or distress;

10 when they did, the experiment was terminated. The bipolar EMG electrodes were

11 connected to a differential amplifier (A-M Systems, Carlsborg, WA; amplification: 10,000x;

12 filter setting: 300-5000 Hz). A ground wire (Ag/AgCl, $0.25 \mathrm{~mm}$ diameter, Medwire, Mt.

13 Vernon, NY) was inserted into the frontal or parietal cortex contralateral to the recording

14 site and a thermocouple was inserted into the frontal or parietal cortex ipsilateral to the

15 recording site. Neurophysiological recordings were performed using a 16-channel silicon

16 electrode or a 4-channel linear probe (A1x16-10mm-100-177; A1x16-8mm-100-177;

17 Q1x4-10mm-50-177, NeuroNexus, Ann Arbor, MI), connected to a data acquisition system

18 (Tucker-Davis Technologies, Alachua, FL) that amplified (10,000x) and filtered (500-5000

$19 \mathrm{~Hz}$ ) the neural signals. A digital interface and Spike2 software (Cambridge Electronic

20 Design, Cambridge, UK) were used to acquire EMG and neurophysiological signals at 1

$21 \mathrm{kHz}$ and at least $12.5 \mathrm{kHz}$, respectively.

22 A micromanipulator ( $\mathrm{FHC}$, Bowdoinham, $\mathrm{ME}$ ) was used to lower the electrode into the

23 brain (DV; IO: 5.5-6.2 mm, LRN: 5-5.8 mm, midbrain structures: 4.5-4.9 mm) until action 
1 potentials were detected. Recording began at least 10 min after multiunit activity (MUA)

2 was detected. Before insertion, the electrode was dipped in fluorescent Dil (Life

3 Technologies, Grand Island, NY) for later identification of the recording sites. Recording

4 of MUA and EMG activity continued for 30 min as the pup cycled freely between sleep and

5 wake (in two pups, activity was recorded for only $15 \mathrm{~min}$ ). The experimenter, blind to the

6 electrophysiological record, scored the pup's sleep and wake behaviors, as described

7 previously (Karlsson et al., 2005).

8 At the end of the recording session, the experimenter assessed evoked neural

9 responses to exafferent stimulation of the limbs. Forelimbs and hindlimbs were gently

10 stimulated using a paint brush. When evoked responses were observed in at least one of

11 the recording channels, stimulation was repeated 20-30 times at intervals of at least 5 s.

12 Each stimulus event was marked using a key press.

14 Histology

15 At the end of all recording sessions, pups were anesthetized with sodium pentobarbital $16(1.5 \mathrm{mg} / \mathrm{g} \mathrm{IP})$ or ketamine/xylazine $(0.02 \mathrm{mg} / \mathrm{g} \mathrm{IP})$ and perfused transcardially with 17 phosphate-buffered saline and 4\% formaldehyde. Brains were sectioned coronally at 80 $\mu \mathrm{m}$ using a freezing microtome (Leica Microsystems, Buffalo Grove, IL). Recording sites were determined by examining Dil tracks, before and after staining with cresyl violet, using

20 a fluorescent microscope (Leica Microsystems, Buffalo Grove, IL).

\section{Retrograde tracing}

23 Retrograde tracing was performed at P8 $(n=9)$ using wheat germ agglutinin (WGA) 
1 conjugated to Alexa Fluor 555 or 488 (Invitrogen Life Technologies, Carlsbad, CA). WGA-

2555 was injected into the IO in 3 pups and into the LRN in 2 pups. In the remaining 2 pups,

3 dual tracing was performed by injecting WGA-488 into the IO and WGA-555 into the LRN.

4 To perform these injections, a pup was anesthetized with $2-5 \%$ isoflurane and secured in

5 a stereotaxic apparatus. A $0.5 \mu$ microsyringe (Hamilton, Reno, NV) was lowered

6 stereotaxically into the IO or LRN and 0.01-0.02 $\mu$ l of 2\% WGA-555 or WGA-488 (dissolved

7 in $0.9 \%$ saline) was injected over $1 \mathrm{~min}$. After a 15 -min post-infusion period, the

8 microsyringe was withdrawn and the incision was closed using Vetbond (3M, Maplewood,

$9 \mathrm{MN})$. The pup was returned to its home cage and perfused $24 \mathrm{~h}$ later as described above.

10 Brains were sectioned coronally at $50 \mu \mathrm{m}$. Every other section was kept for Nissl staining

11 for verification of the injection sites and areas that show retrograde labeling. Retrogradely

12 labeled cell bodies were imaged using a fluorescent microscope (DFC300FX, Leica, Buffalo

13 Grove, IL)

14

\section{Stimulation of MDJ structures}

16 In urethanized $(1.5 \mathrm{mg} / \mathrm{g})$ head-fixed $\mathrm{P} 8$ rats $(\mathrm{n}=8)$, a parylene-coated tungsten stimulating

17 electrode (World Precision Instruments, Inc., Sarasota, FL) was lowered into the MDJ

18 nuclei most strongly implicated by retrograde tracing. The nuclei were electrically

19 stimulated to produce discrete movements of the forelimbs and/or hindlimbs. Trains of

20 pulses (pulse duration: 0.2-0.4 ms; pulse frequency: $300 \mathrm{~Hz}$; train width: $45 \mathrm{~ms}$; Williams

21 et al., 2014) were delivered every $5 \mathrm{~s}$ for $60 \mathrm{~min}$. The current was adjusted (300-900 $\mu \mathrm{A})$

22 as needed to ensure that stimulation continued to reliably produce movement. Ninety min 
1 after the last stimulation, the pup wa sacrificed and the brain was prepared for c-Fos

2 immunohistochemistry.

\section{Immunohistochemistry for c-Fos expression}

$5 \quad$ Brains were sliced in $50 \mu \mathrm{m}$ sections and every other section was kept for Nissl staining

6 for verification of the stimulation sites and visualization of c-Fos expression, respectively.

7 Primary antibody against c-Fos (anti-c-Fos rabbit polyclonal IgG; Santa Cruz

8 Biotechnology) was diluted $1: 1000$ in a universal blocking serum (2\% bovine serum

9 albumin; $1 \%$ triton; $0.02 \%$ sodium azide) and applied to the sections. Sections were

10 coverslipped and left to incubate for $48 \mathrm{~h}$ at $4^{\circ} \mathrm{C}$. After incubation of the primary antibody

11 and a series of washes in PBS, a secondary antibody (Alexa Fluor 488 donkey anti-rabbit

12 IgG; Life Technologies, Grand Island, NY; 1:500 in PBS) was applied to the section and

13 incubate for $90 \mathrm{~min}$ at room temperature. The slides were coverslipped using Fluoro-Gel

14 (Electron Microscopy Sciences (Hatfield, PA) and expression of c-Fos was examined 15 using a fluorescent microscope (DFC300FX or DM6B, Leica, Buffalo Grove, IL).

\section{Intra-IO injection of apamin}

In 18 P8 rats, pups were prepared for electrophysiological recording as described above and transferred to the recording rig. Once a pup started cycling between sleep and wake,

a $0.5 \mu \mathrm{l}$ microsyringe was lowered stereotaxically into the $\mathrm{IO}$ and $100 \mathrm{nl}$ of apamin.

21 (Abcam, Cambridge, MA; $1 \mu \mathrm{M}$, dissolved in $0.9 \%$ saline, $n=8)$ or saline $(n=10)$ was

22 injected over $1 \mathrm{~min}$. During preparation of the drug or vehicle, fluorogold $(4 \%$,

23 Fluorochrome, Denver, CO) was added to the solutions for subsequent assessment of the 
1 extent of drug diffusion. After a 15-min period to allow for diffusion, the microsyringe was

2 withdrawn and a recording electrode was lowered in its place into the $\mathrm{IO}$ and activity was

3 recorded for $30 \mathrm{~min}$. At the end of the experiment, the pup was sacrificed and its brain was

4 prepared for histology as described above.

6 Data analysis

7 Spike sorting. As described previously (Mukherjee et al., 2017; Sokoloff et al., 2015a),

8 action potentials (signal-to-noise $\geq 2: 1$ ) were sorted from MUA records using template

9 matching and principal component analysis in Spike2 (Cambridge Electronic Design).

10 Waveforms exceeding 3.5 SD from the mean of a given template were excluded from

11 analysis.

Identification of behavioral states. EMG activity and behavioral scoring were used to identify behavioral state (Blumberg et al., 2015). To establish an EMG threshold for distinguishing sleep from wake, EMG signals were rectified and smoothed (tau $=0.001 \mathrm{~s}$ ).

15 The mean amplitude of high muscle tone and atonia were calculated from five representative 1-s segments and the midpoint between the two was used to establish the

17 threshold for defining periods of wake (defined as muscle tone being above the threshold for at least $1 \mathrm{~s}$ ) and sleep (defined as muscle tone being below the threshold for at least 1 s). Active wake (AW) was identified by high-amplitude limb movements (e.g., stepping,

20 stretching) against a background of high muscle tone and was confirmed using behavioral 21 scoring. The onset of a wake movement was defined on the basis of EMG amplitude 22 surpassing the established threshold. Active sleep (AS) was characterized by the 23 presence of myoclonic twitches of the limbs against a background of muscle atonia. 
1 Twitches were identified as sharp EMG events that exceeded by $\geq 3 x$ the mean EMG

2 baseline during atonia; twitches were also confirmed by behavioral scoring (Seelke and

3 Blumberg, 2010). Additionally, behavioral quiescence (BQ) was characterized as periods

4 of low muscle tone interposed between AW and AS.

6 states was determined. Bouts of $A S, A W$, and $B Q$ were excluded when firing rates

7 exceeded 3 SD of the firing rate for that behavioral state; this happened rarely $(0-2$ per

8 unit). Next, pairwise comparison of firing rates across states was performed using the

9 Wilcoxon matched-pairs signed-ranks test (SPSS; IBM, Armonk, NY). Units were

categorized as AS-on ( $A S>A W \geq B Q), A W$-on $(A W>A S \geq B Q), A S+A W$-on $(A S=A W>$

$11 \mathrm{BQ})$ or state-independent $(\mathrm{AS}=\mathrm{AW}=\mathrm{BQ})$. Firing rates of all AS-on units across behavioral

states were further compared using the Wilcoxon matched-pairs signed-ranks test.

Twitch-triggered neural activity. To determine the relationship between unit activity and

twitching, we triggered unit activity on twitch onsets and generated perievent histograms

over a 1-s window using 5- or 10-ms bins. We performed these analyses on each individual unit using twitches from nuchal, forelimb, and hindlimb muscles. We tested statistical significance by jittering twitch events 1000 times over a 500-ms window using PatternJitter

18 (Amarasingham et al., 2012; Harrison and Geman, 2009). Then using a custom-written

Matlab program (MathWorks, Natick, MA), we generated upper and lower confidence bands ( $p<0.05$ or 0.01 for each confidence band) using a method that corrects for multiple comparisons (Amarasingham et al., 2012). For each unit, after histograms were separately constructed for nuchal, forelimb, or hindlimb twitches, we identified and activity that was

23 significant in response to a twitch. When more than one muscle yielded a significant 
1 change in neural activity, we further analyzed the data only for the muscle that showed the

2 strongest relationship (determined by the highest firing rate) between twitches and unit

3 activity. We then pooled these data to create perievent histograms composed of significant

4 units and performed jitter analyses on the pooled data.

Wake-triggered neural activity. To determine the relationship between neural activity

6 and wake movements, we triggered unit activity on wake-movement onset and created

7 perievent histograms on the pooled data (20-ms bins, 1 -s window). We then performed

8 jitter analysis as described above.

9 Evoked response to exafferent stimulation. We identified MUA in which evoked responses were observed and then sorted the units. Those units were then pooled and

11 triggered on stimulus onset (determined using EMG artifact) to create perievent histograms. The jitter analysis was performed on the pooled data, as described above. behavior. We assessed the amount of time spent in AS and the number of twitches per min of AS in each pup. Differences across groups were tested using the Mann-Whitney $U$ test. Next, we determined if apamin altered the overall firing rate. We calculated the firing rate of each unit during AS and compared that across groups using the Mann-Whitney $U$ test. One value exceeding 3 SD was excluded as an outlier.

We then assessed whether apamin altered the shape of twitch-triggered perievent histogram. First, we created perievent histograms (10-ms bins, 1-s window) for each unit

21 as described above. For each unit, firing rate was normalized to the peak firing rate and 22 the average normalized firing rate across all units in each group was calculated. Perievent 23 histograms were then created with the average (+SEM) normalized firing rates triggered 
1 on twitches for each group. Next, we assessed how apamin altered the pattern of twitch-

2 triggered activity of individual units. To do that, we identified significant units by performing

3 jitter analysis on individual units as described above. We counted the percentage of units

4 that showed precise peak within \pm 10 ms around a twitch and compared that across groups

5 using a Chi-squared test. Finally, we pooled significant units in each group and pooled

6 them to create perievent histograms consisting of significant units only. To assess the

7 difference in the shape of perievent histograms, we calculated the area under the curve

8 by adding the histogram counts within a particular time window and compared that across

9 groups using the Mann-Whitney $U$ test. One value exceeding 3 SD was excluded as an

10 outlier.

11 Unless otherwise stated, alpha was set at 0.05 . 


\section{FIGURES AND LEGENDS}

A

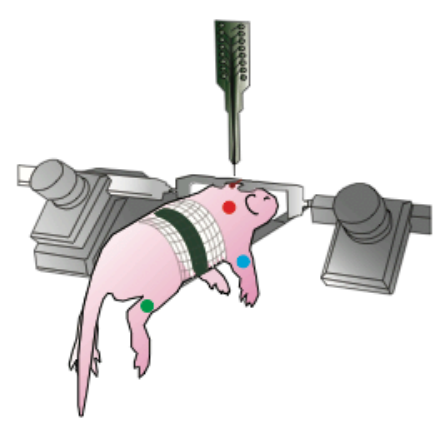

B

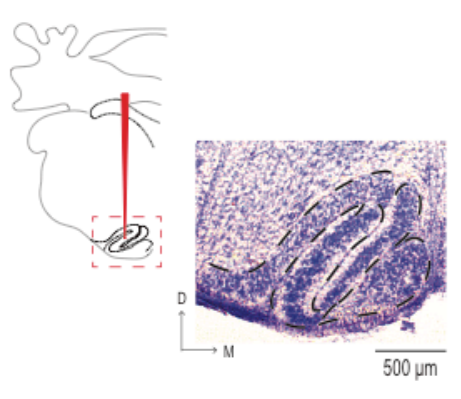

E
State dependency

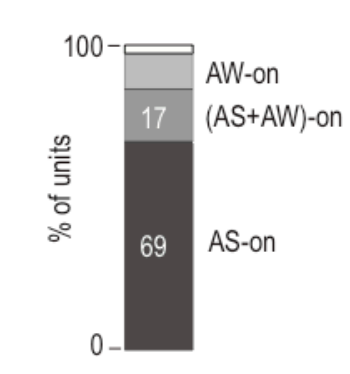

C

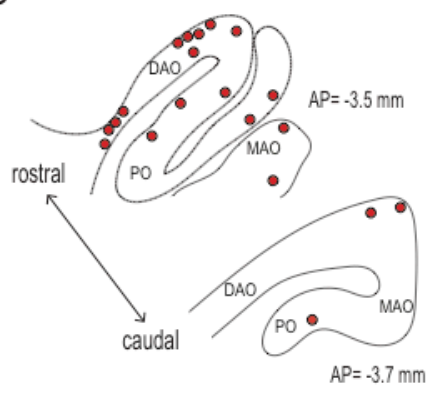

$\mathrm{F}$

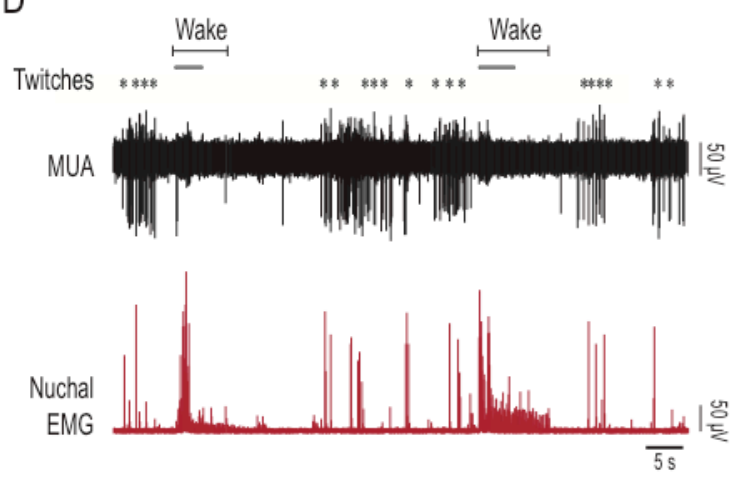

AS-on units

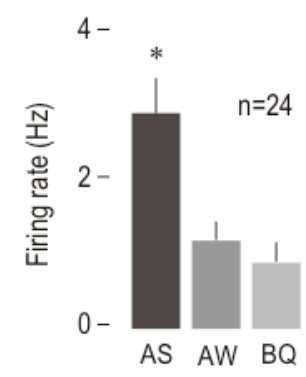

Figure 1. Olivary activity predominates during active sleep. (A) Illustration of a headfixed rat pup in a recording apparatus instrumented with nuchal (red), forelimb (blue), and hindlimb (green) EMG electrodes. (B) Left: Reconstruction of a representative electrode placement within the IO (red vertical line). Red dashed line circumscribes the IO. Right: Coronal section stained with cresyl violet depicting the anatomical location of the IO (black dashed line). (C) Reconstruction of electrode placements (red circles) within the IO in two coronal sections across all subjects. (D) Representative recording of rectified nuchal EMG activity and multiunit activity (MUA) in the 10 during spontaneous sleep-wake cycling. Asterisks denote twitches and gray horizontal bars denote wake movements as scored by the experimenter. (E) Stacked plot depicting the percentage of units that were AS-on, (AS+AW)-on, and AW-on units in the IO. (F) Bar graphs showing mean (+SEM) firing rates of AS-on units across behavioral states. * significant difference from $A W$ and $B Q$, $p<0.0005$. $D=$ dorsal; $M=$ medial; $D A O=$ dorsal accessory olive; $M A O=$ medial accessory olive; $\mathrm{PO}=$ principal olive; $\mathrm{AP}=$ antero-posterior in relation to lambda; $\mathrm{AS}=$ active sleep; $A W=$ active wake; $B Q=$ behavioral quiescence. 


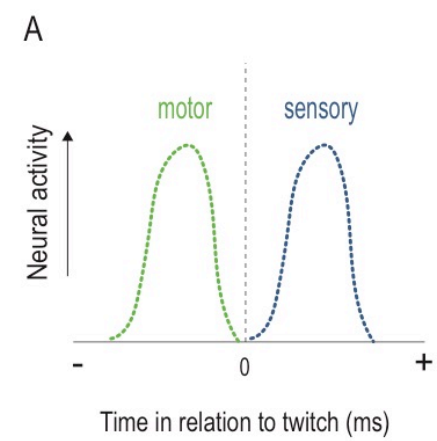

B

C
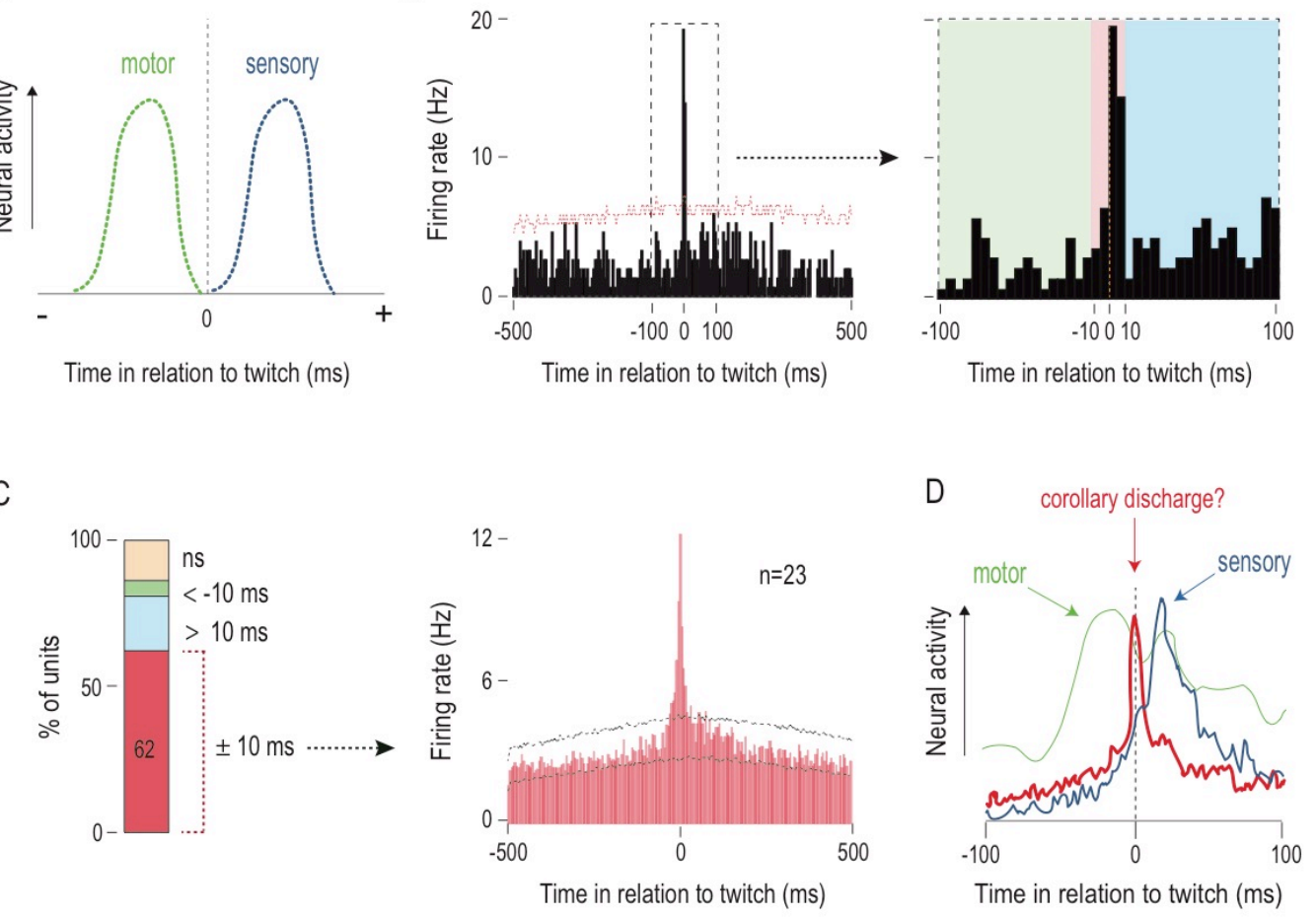

Figure 2 and 1 supplement. Twitches trigger sharp, short-latency olivary activity. (A) Perievent histograms for idealized motor and sensory units in relation to twitch onset. Motor activity precedes the onset of twitches (green line) and reafference follows the onset of twitches (blue line). Black vertical line denotes twitch onset. (B) Left: Perievent histogram (5-ms bins) showing sharp, short-latency activity of a representative $1 \mathrm{O}$ unit in relation to nuchal muscle twitches. Upper confidence band ( $p<0.01$ for each band) is indicated by red horizontal dashed line (lower confidence band is at zero). Black dashed box demarcates the \pm 100 -ms time window around twitches. Right: The \pm 100 -ms time window around twitches at left is shown. Green, red, and blue shaded areas denote $<-10-$ $\mathrm{ms}, \pm 10$-ms, and $>10$-ms time windows, respectively. (C) Left: Stacked plot showing the percentage of $1 \mathrm{O}$ units that exhibited significant increases in firing within \pm 10 -ms (red), $>10-\mathrm{ms}$ (blue), and <-10-ms (green) time windows around twitches. Right: Perievent histogram showing 10 unit activity in relation to twitches for those units that were significantly active in the \pm 10 -ms time window. Data are pooled across 23 units and triggered on 6,602 twitches. Upper and lower confidence bands ( $<<0.01$ for each band) are indicated by black and gray horizontal dashed lines, respectively. (D) Comparison of $1 \mathrm{O}$ activity in relation to twitches (red; from $\mathrm{C}$ ) with that of a representative motor (green line; data from Del Rio-Bermudez et al., 2015) and sensory (blue line; data from Tiriac and Blumberg, 2016) structure. Black vertical dashed line denotes twitch-onset. 
A

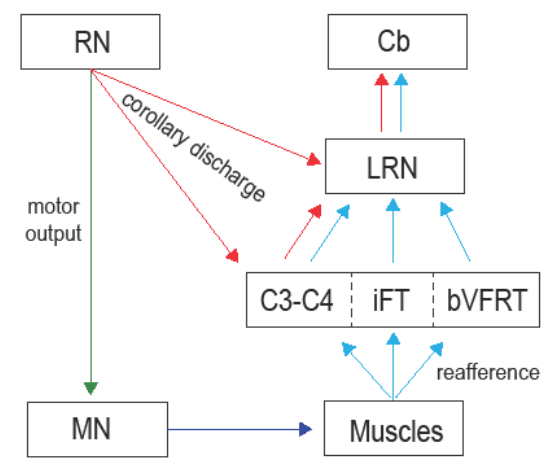

C

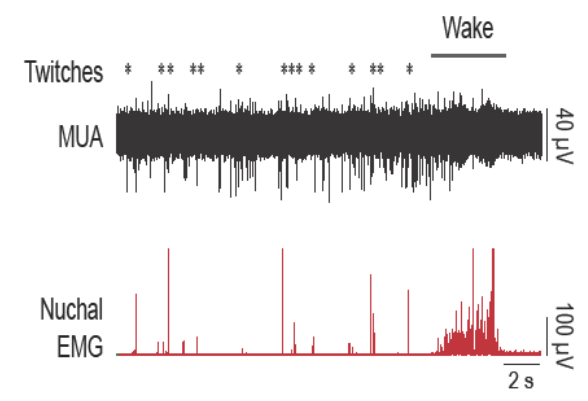

G

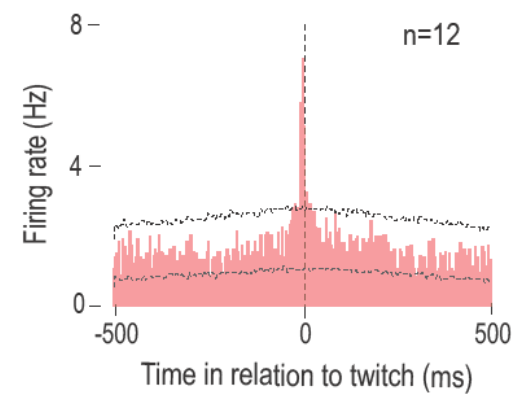

B
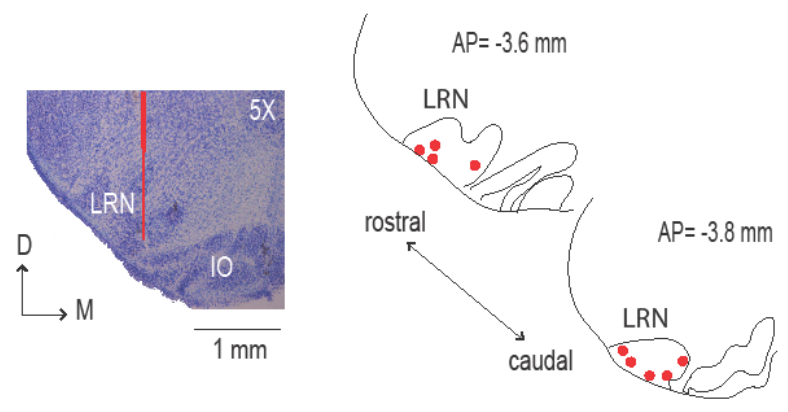

$E$

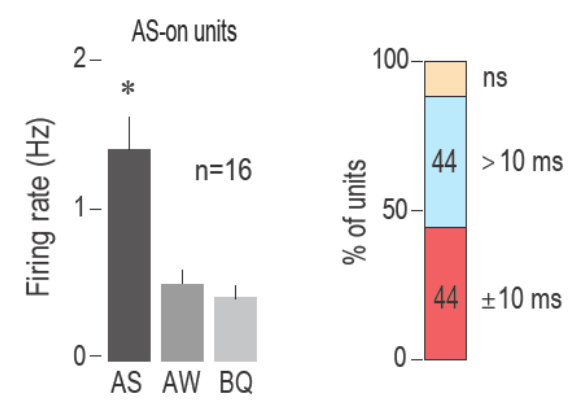

$\mathrm{H}$
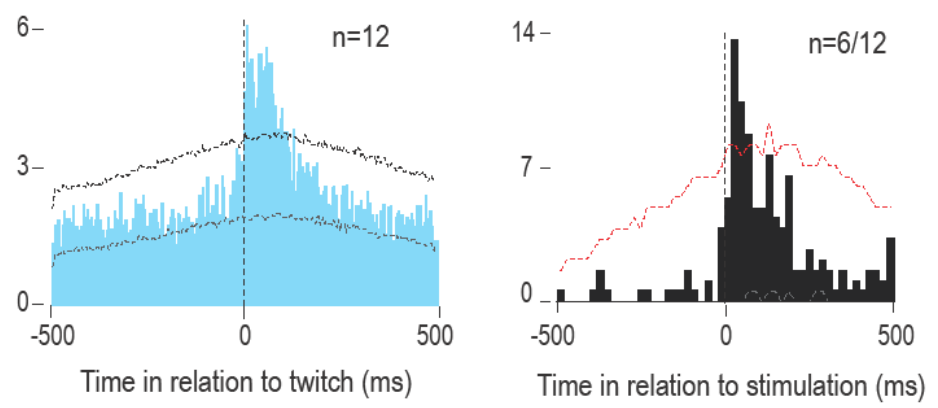

Figure 3. The LRN receives twitch-related corollary discharge and reafference signals. (A) Diagram depicting afferent and efferent connections of the LRN. Pathways conveying motor commands (green), reafference (blue), and corollary discharge (red) are shown (see Alstermark and Ekerot, 2013). (B) Left: Coronal section (5x) stained with cresyl violet depicting the anatomical location of the LRN in the brainstem and reconstruction of a representative electrode placement (red vertical line) in the LRN. Right: Reconstruction of electrode placements (red circles) within the LRN in two coronal sections across all P8 subjects $(n=9)$. (C) Representative recording of rectified nuchal EMG activity and multiunit activity (MUA) in the LRN during spontaneous sleep-wake cycling. Asterisks denote twitches and gray horizontal bars denote wake movements as scored by the experimenter. 
(D) Stacked plot showing the percentage of AS-on, (AS+AW)-on, and AW-on units in the LRN. (E) Bar graphs showing mean (+SEM) firing rates of AS-on units across behavioral states. * significant difference from $A W$ and $B Q, p<0.0005$. (F) Stacked plot depicting the percentage of units that significantly increased their firing rates \pm 10 ms around twitches (red) and >10 ms following twitches (blue). (G) Perievent histograms (5-ms bins) showing LRN unit activity in relation to twitches. Left: Data pooled across 12 units (and triggered on 3,688 twitches) that significantly increased their activity in the \pm 10 -ms time window (red). Right: Data pooled across the 12 units (and triggered on 5264 twitches) that exhibited a significant peak with a latency of $>10 \mathrm{~ms}$. Black vertical dashed lines indicate twitch onset. Upper and lower confidence bands ( $p<0.01$ for each band) are indicated by black and green horizontal dashed lines, respectively. (H) Perievent histogram (20-ms bins) depicting LRN unit activity ( $n=6$ of a possible total of 12) in response to forelimb or hindlimb stimulation for those units that significantly increased their activity in the $>10 \mathrm{~ms}$ time window (blue histogram in $\mathrm{G}$ ). Black vertical dashed line corresponds to stimulation onset determined using EMG activity. Upper confidence band $(p<0.05)$ is indicated by red horizontal dashed line. RN: red nucleus; MN: motor neurons; C: cervical segment; LRN: lateral reticular nucleus; $\mathrm{Cb}$ : cerebellum; IO: inferior olive; D: dorsal; M: medial; AP: anteroposterior in relation to lambda; $A S$ : active sleep; $A W$ : active wake; $B Q$ : behavioral quiescence; ns: not significant. 
A

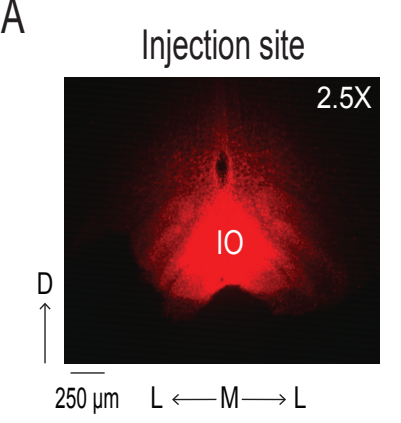

B

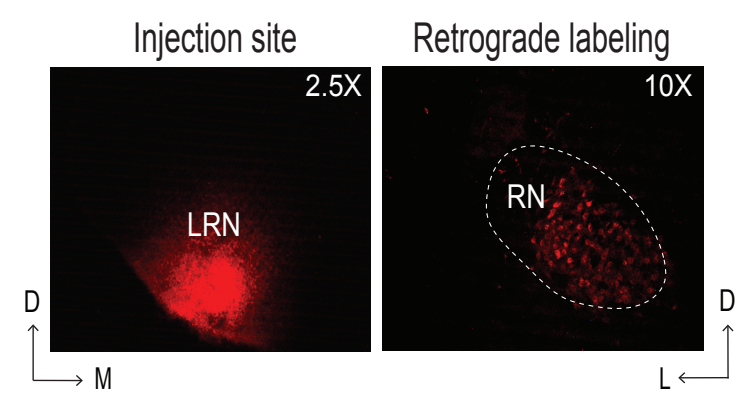

Retrograde labeling

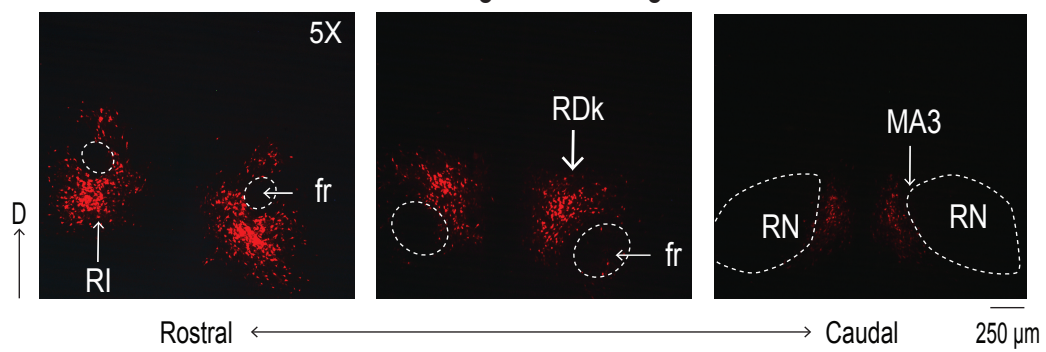

C Injection sites Retrograde labeling

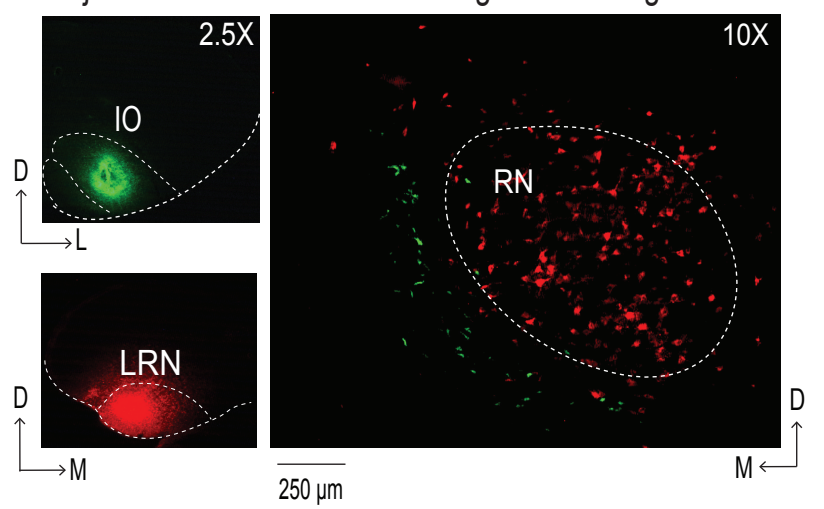

Figure 4 and 1 supplement. Retrograde labeling of the mesodiencephalic junction (MDJ) after infusion of WGA into the IO and LRN of P8 rats. (A) Left: Coronal section (2.5x) depicting WGA-555 diffusion in the IO. Right: Coronal sections $(5 x)$ depicting retrogradely labeled cell bodies in multiple MDJ nuclei. (B) Left: Coronal section (2.5x) depicting WGA-555 diffusion in the LRN. Right: Coronal section (10x) depicting retrogradely labeled cell bodies in the red nucleus (RN). (C) Left: Coronal sections (2.5x) depicting WGA-488 diffusion in the IO (top; green) and WGA-555 diffusion in the LRN (bottom; red) in the same P8 rat. Right: Coronal section (10x) depicting retrogradely labeled cell bodies in the RN (red; from LRN) and the region immediately medial to it (green; from IO). D: dorsal; L: lateral; M: medial; IO: inferior olive; RI: rostral interstitial nucleus of Cajal; fr: fasciculus retroflexus; RDk: rostral nucleus of Darkschewitsch; MA3: accessory oculomotor nuclei; LRN: lateral reticular nucleus. 
A

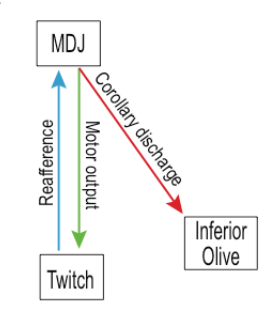

D

Twitch-related activity

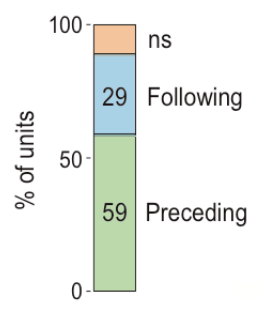

B

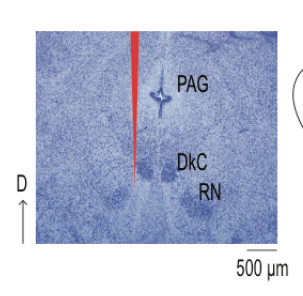

$\mathrm{E}$

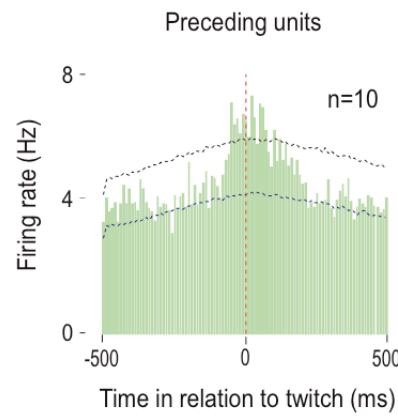

C

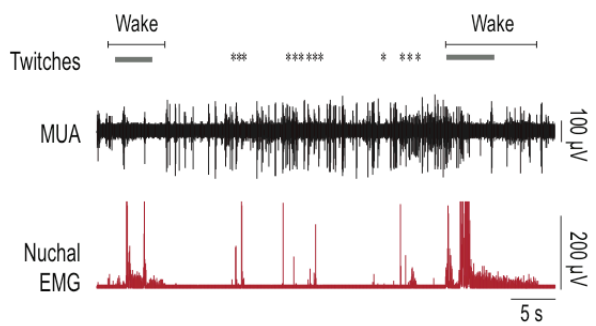

$\mathrm{F}$
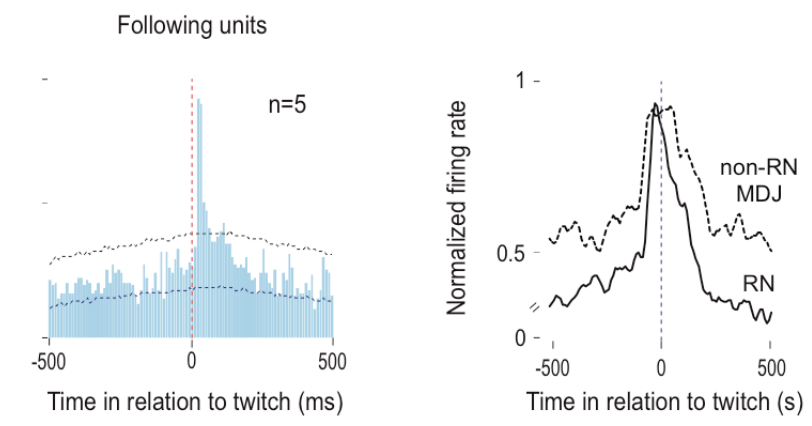

Figure 5. MDJ structures adjacent to the red nucleus exhibit twitch-preceding and twitch-following activity. (A) Diagram showing anatomical connections of the MDJ regions that lie adjacent to the red nucleus. Proposed pathways conveying motor commands (green line), reafference (blue line), and corollary discharge (red line) are shown. (B) Left: Reconstruction of a representative electrode placement (red vertical line; 2.5x). Right: Reconstruction of electrode placements (red circles) in the MDJ in two coronal sections across all pups $(n=7)$. (C) Representative recording of rectified nuchal EMG activity and multiunit activity (MUA) in the MDJ during spontaneous sleep-wake cycling. Asterisks denote twitches and gray horizontal bars denote wake movements as scored by the experimenter. (D) Stacked plot depicting the percentage of twitch-preceding (motor; green) and twitch-following (sensory; blue) units in the MDJ. (E) Left: Perievent histogram (10-ms bins) showing activity of twitch-preceding MDJ units in relation to twitches. Data pooled across 10 units and triggered on 2877 twitches. Right: Perievent histograms (10ms bins) showing activity of twitch-following MDJ units in relation to twitches. Data pooled across 5 units and triggered on 1382 twitches. Red vertical dashed lines correspond to twitch onset. Upper and lower confidence bands ( $p<0.05$ for each band) are indicated by black and blue horizontal dashed lines, respectively. (F) Perievent histograms (10-ms bins) comparing normalized firing rate in relation to twitch onset for twitch-preceding units in the red nucleus (RN; black solid line; data from Del Rio-Bermudez et al., 2015) with that of non-RN MDJ units adjacent to the red nucleus (black dashed line; redrawn from $E$, left). Black vertical dashed lines correspond to twitch onsets. D: dorsal; PAG: periaqueductal gray; DkC: caudal nucleus of Darkschewitsch; ns: not significant. 
A

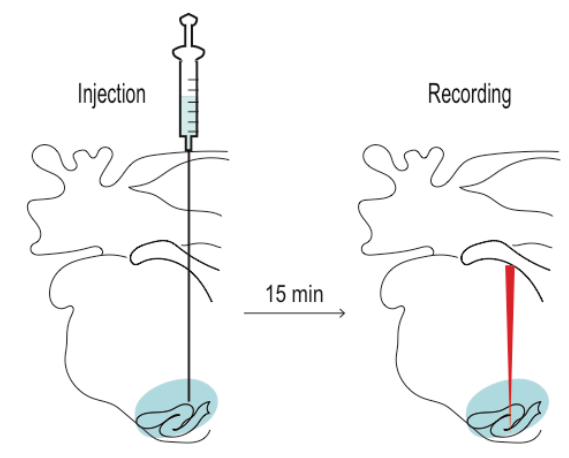

C

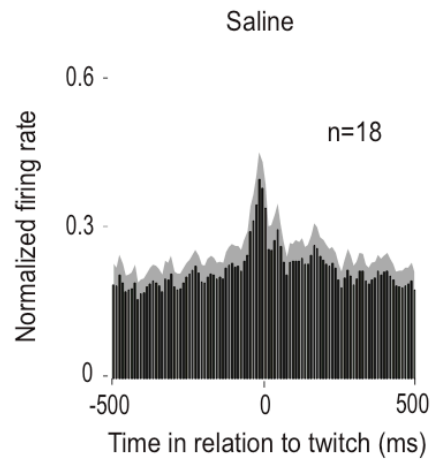

$\mathrm{E}$

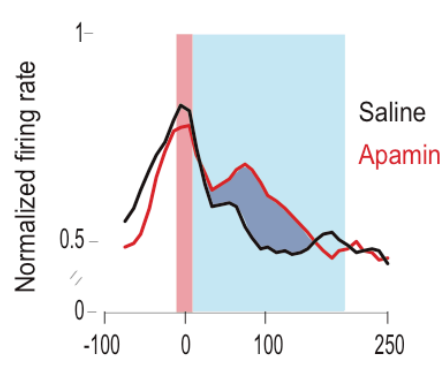

Time in relation to twitch (ms)
B
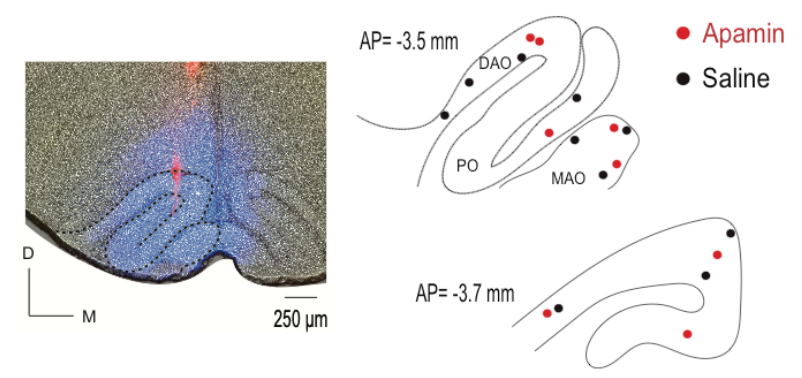

D

Apamin
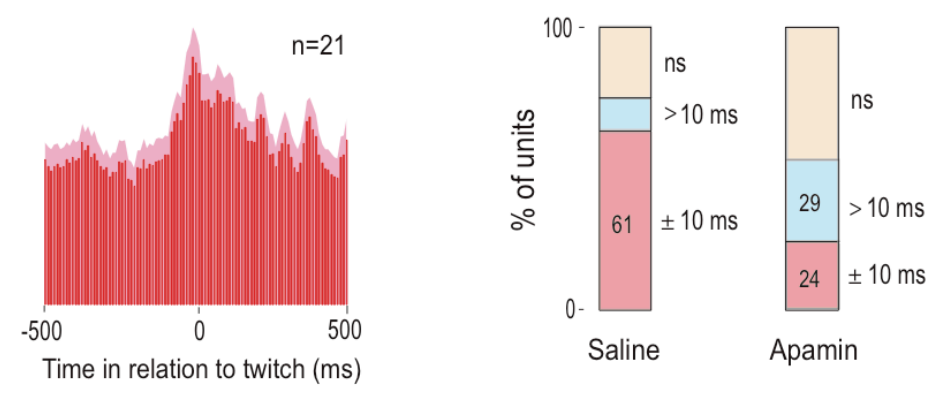

G

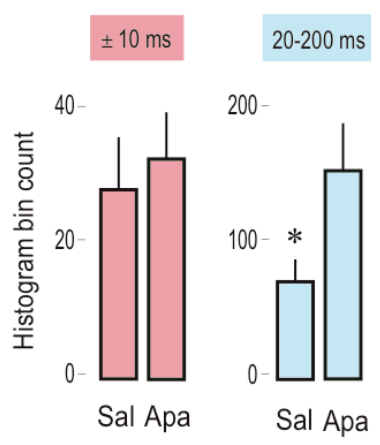

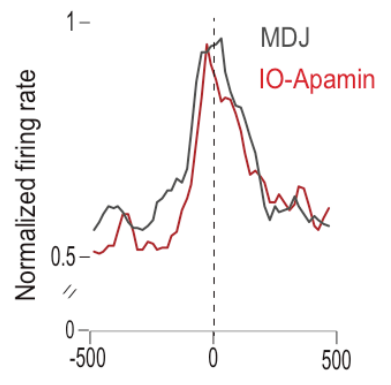

Time in relation to twitch (ms)

Figure 6 and 1 supplement. Apamin broadens the twitch-related peak in the IO. (A) Diagram depicting experimental design. Apamin or saline, mixed with $4 \%$ Fluorogold, was microinjected into the 10 . Blue shaded area shows the extent of drug diffusion. Fifteen minutes after the injection, the microsyringe was withdrawn and a recording electrode, coated with Dil (red vertical line), was inserted into the IO. Unit activity was recorded for 30 min. (B) Left: Representative coronal section showing drug diffusion in the 10 (blue shaded area) and placement of recording electrode (red) within the IO. Right: Reconstruction of electrode placements within the 10 in two coronal sections for all pups 
in the saline (black dots; $n=10$ pups) and apamin (red dots; $n=8$ pups) groups. (C) Perievent histograms (10-ms bins) showing mean (+SEM) normalized firing rate across all units triggered on twitches in the saline $(n=18)$ and apamin $(n=21)$ groups. (D) Stacked plots showing the percentage of units with significant activity within \pm 10 ms around twitches (red) and $>10 \mathrm{~ms}$ following twitches (blue) in the saline and apamin groups. (E) Perievent histograms (10-ms bins) showing 10 unit activity in relation to twitches in the saline (black line) and apamin (red line) groups. Data are pooled across significant units only (red + blue stacks in E) in both groups ( $n=13$ in saline and $n=11$ in apamin group) and smoothed (tau=10 ms). Red shaded area denotes $\pm 10-\mathrm{ms}$ time window around twitches. Blue shaded area denotes 20-200-ms time window following twitches. (F) Bar graphs showing the area under the curve (mean + SEM) during two time windows: the $\pm 10-m s$ window around twitches (red) and the 20-200-ms window following twitches (blue) for the units in the saline $(n=12)$ and apamin $(n=11)$ groups. * $p=0.03$. (G) Perievent histograms comparing normalized firing rate in relation to twitch onset for twitch-preceding units in the non-RN MDJ (gray line, taken from Figure 5E) with that of 10 units in the apamin group (red line). DAO: dorsal accessory olive; MAO: medial accessory olive; PO: principal olive; D: dorsal; M: medial; AP: antero-posterior in relation to bregma; sal: saline; apa: apamin; ns: not significant. 
A. Twitches

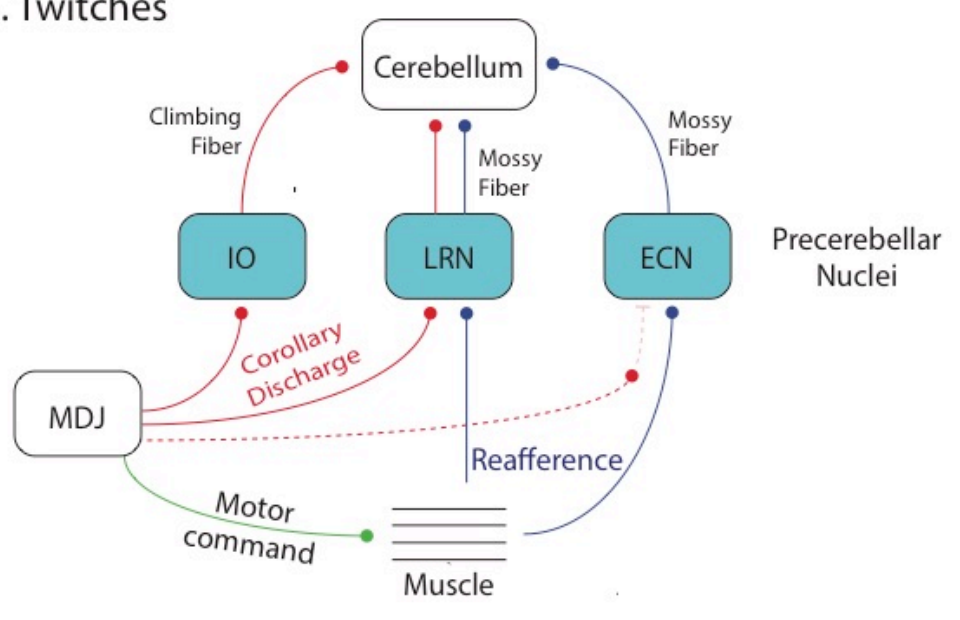

B. Wake movements

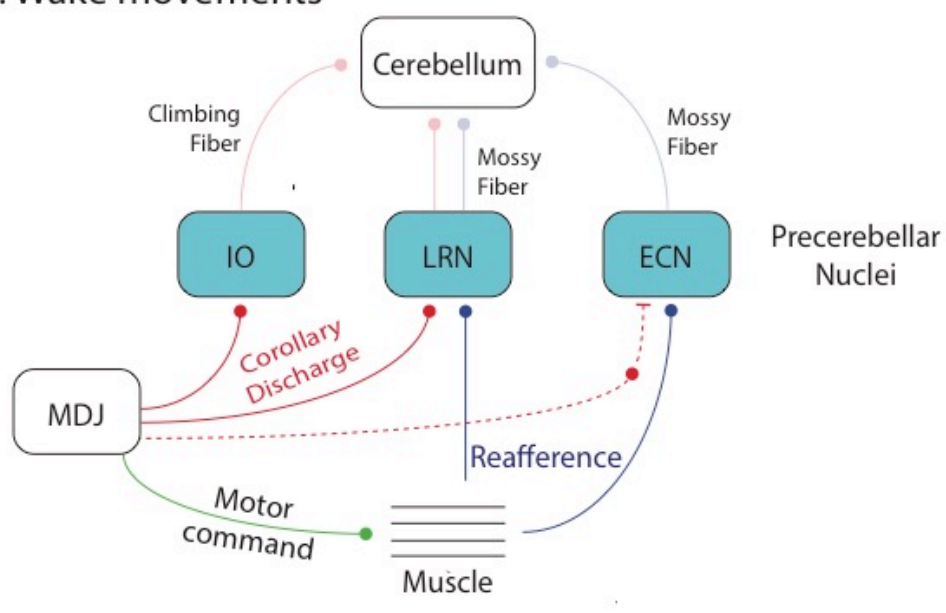

Figure 7. Summary diagrams depicting flow of neural activity in the cerebellar system in association with twitches and wake movements in week-old rats. (A) Flow of twitch-related CD from the MDJ to the IO and LRN and reafference from the limbs to the LRN and ECN. Both CD and reafference converge in the cerebellum via climbing and mossy fibers. Dotted lines denote hypothesized functional connections. (B) Activity is less pronounced or actively suppressed during wake movements in these three precerebellar nuclei, thereby reducing input to the cerebellum. 
A

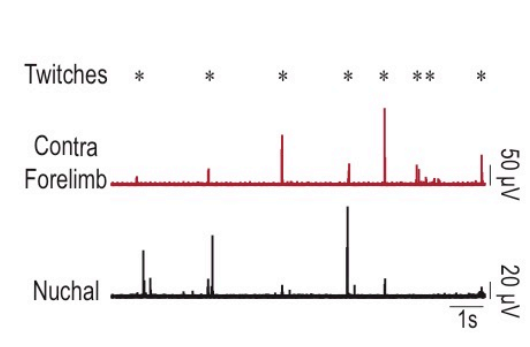

B
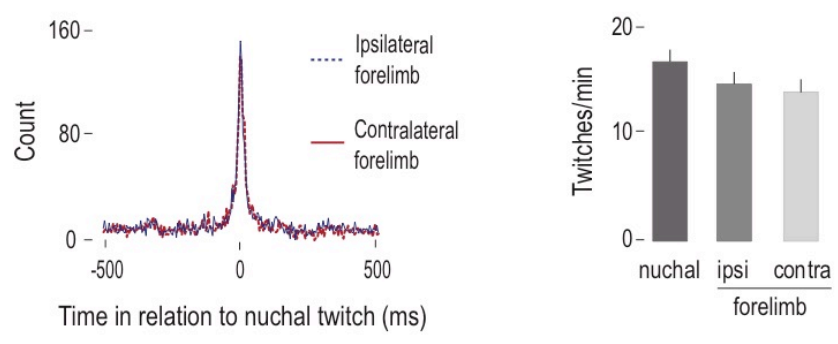

C

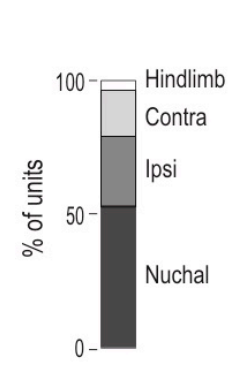

D
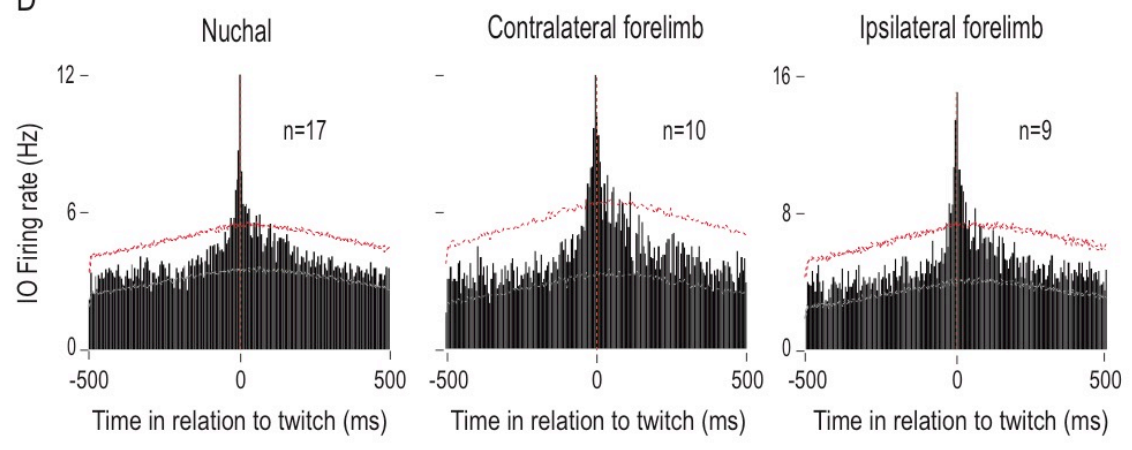

E

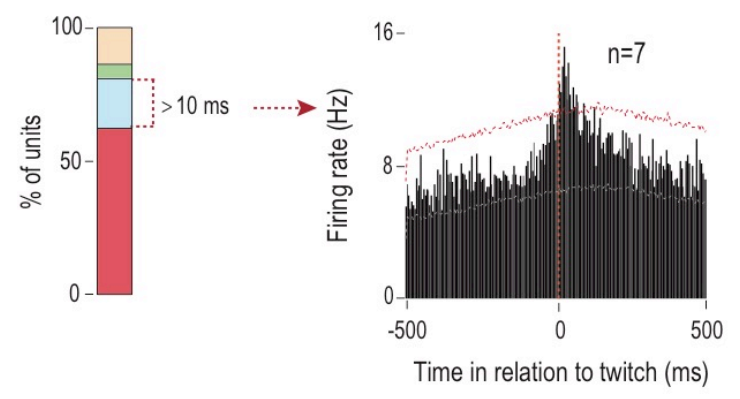

Figure 2-figure supplement 1. IO units respond predominantly to nuchal and forelimb twitches. (A) Left: Representative recording of nuchal and contralateral forelimb EMG activity showing co-occurrence of twitches in those muscles. Asterisks denote experimenter-scored twitches. Right: Cross-correlogram (5-ms window) depicting cooccurrence of nuchal muscle twitches with contralateral (red line) and ipsilateral (blue dotted line) forelimb twitches. (B) Bar graphs depicting mean (+SEM) rates of twitching in nuchal muscle and ipsilateral and contralateral forelimb muscles. Data are pooled across 15 pups (C) Stacked plot showing the percentage of 10 units that exhibited sharp, zerolatency activity in relation to nuchal, ipsilateral forelimb, contralateral forelimb, and hindlimb twitches. (D) Perievent histograms showing $1 \mathrm{O}$ unit activity in relation to nuchal muscle and contralateral and ipsilateral forelimb twitches. Data are pooled across 17, 10, and 9 units, respectively. Upper and lower confidence bands ( $p<0.01$ for each band) are 
indicated by red and gray horizontal dashed lines, respectively. ipsi: ipsilateral; contra: contralateral. (E) Left: Stacked plot showing percentages of $I O$ units that exhibited significant increase in firing in $>10$-ms (blue stack) time windows around twitches. Right: Perievent histograms showing 10 unit activity in relation to twitches. Data pooled across 7 units. Ipsi=ipsilateral; contra=contralateral; ns=not significant. 
A

\section{Stimulation sites}

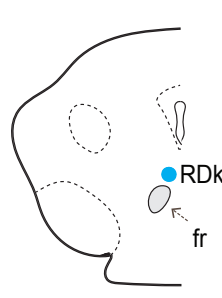

rostral

C

LRN c-Fos expression

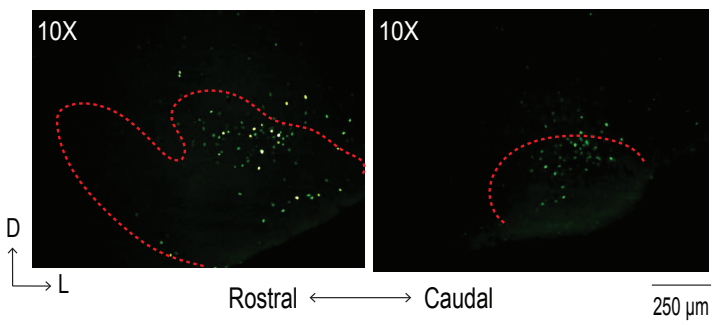
caudal
B

10 c-Fos expression
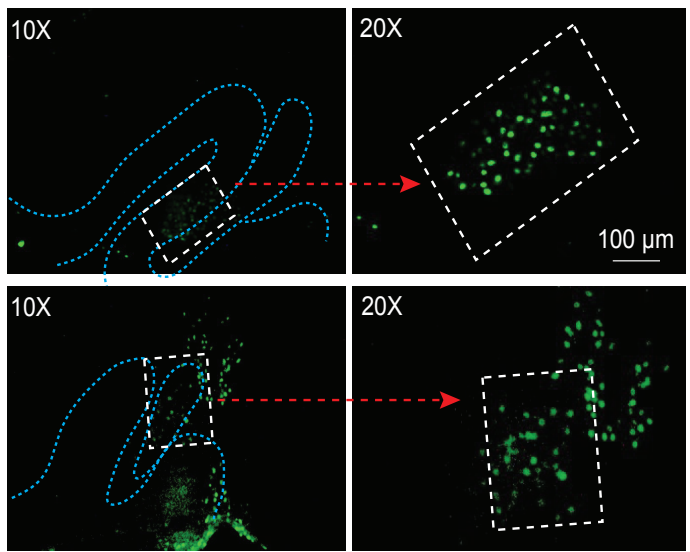

$20 \mathrm{X}$
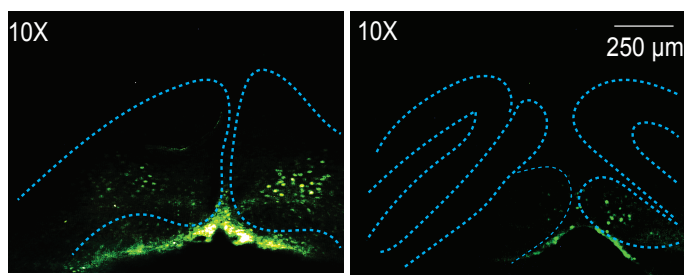

Figure 4-figure supplement 1. (A) Reconstruction of stimulation sites inside (red dots) and adjacent to (blue dots) the red nucleus (RN) in three coronal sections. (B) Representative photomicrographs in two different magnifications showing c-Fos expression in three sections of the 10 (demarcated by blue dotted lines). (C) Representative photomicrographs showing c-Fos expression in two sections of the LRN (demarcated by red dotted lines). RDk: rostral nucleus of Darkschewitsch; fr: fasciculus retroflexus; MA3: accessory oculomotor nucleus; Dk: nucleus Darkschewitsch; $D=d o r s a l ;$ $\mathrm{L}=$ lateral. 
non-RN MDJ

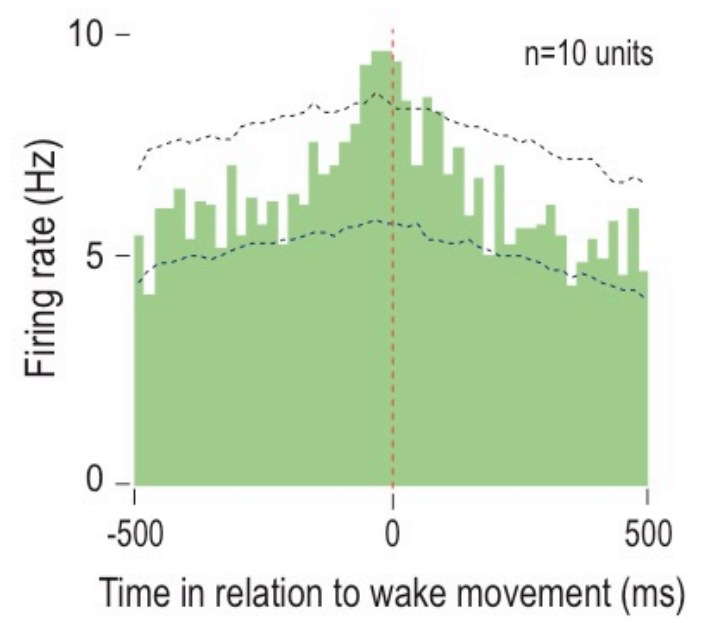

Inferior olive

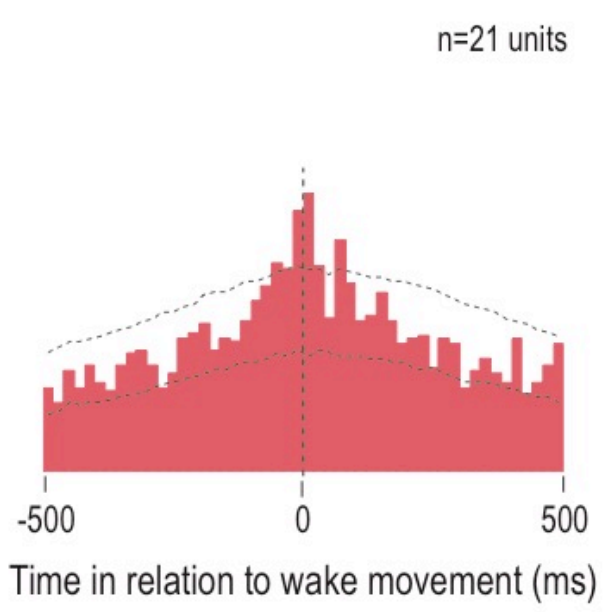

Figure 5-figure supplement 1. (A) Perievent histogram showing activity of twitchpreceding MDJ units (outside the RN) in relation to wake movements. Data pooled across 10 units and triggered on 586 wake movements. (B) Perievent histogram showing 10 unit activity in relation to wake movements for those units that were significantly active in the \pm 10 -ms time window around a twitch. Data pooled across 21 units and triggered on 747 wake movements. 
A

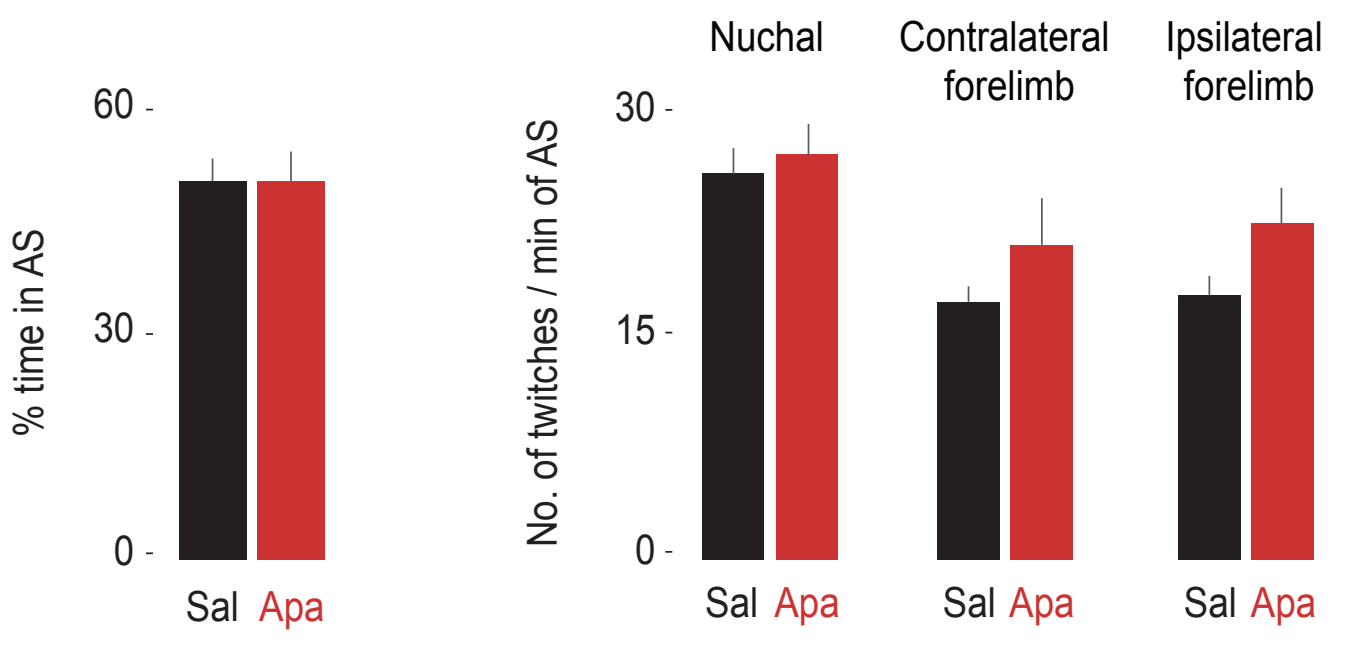

C

Saline

Apamin

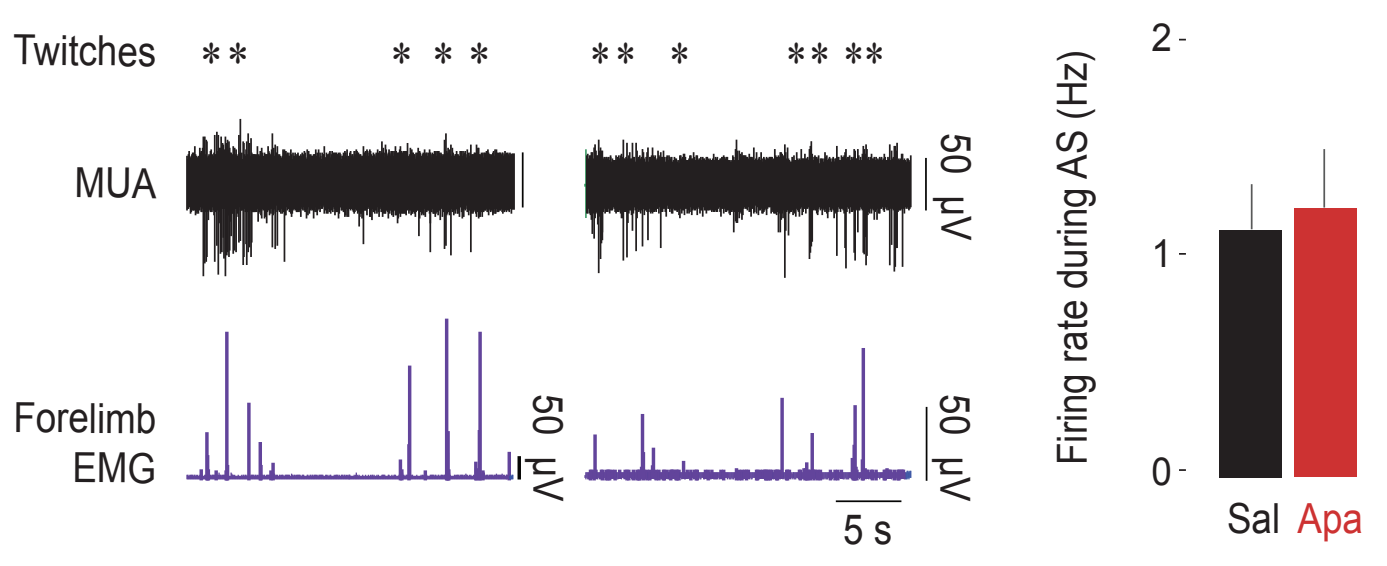

Figure 6-figure supplement 1. Apamin does not affect sleep-wake behavior. (A) Bar graphs showing the mean (+SEM) percentages of time spent in active sleep in saline ( $n=10$ pups) and apamin groups ( $n=8$ pups). (B) Bar graphs showing the mean (+SEM) rates of nuchal, contralateral and ipsilateral forelimb twitching in saline ( $n=10$ pups) and apamin ( $n=8$ pups) groups. (C) Representative recording of rectified forelimb EMG activity, and MUA in the 10 during spontaneous sleep-wake cycling in saline (left) and apamin (right) groups. Asterisks denote twitches and gray horizontal bars denote wake movements as scored by the experimenter. (D) Bar graphs showing the mean (+SEM) firing rates of units during AS in saline ( $n=18$ units) and apamin groups ( $n=22$ units). Sal=saline; Apa=apamin. 


\section{ACKNOWLEDGMENTS}

The authors thank Alex Tiriac and Jimmy Dooley for helpful comments. Research was supported by grants from the National Institute of Health (R37 HD-081168 and R01 HD063071) to MSB. The authors declare no competing financial interests. 


\section{REFERENCES}

Akhmetshina D, Nasretdinov A, Zakharov A, Valeeva G, and Khazipov R. The nature of the sensory input to the neonatal rat barrel cortex. J Neurosci 36: 9922-9932, 2016.

Alstermark B, and Ekerot CF. The lateral reticular nucleus: a precerebellar centre providing the cerebellum with overview and integration of motor functions at systems level. A new hypothesis. J Physiol 591: 5453-5458, 2013.

Alstermark B, and Ekerot CF. The lateral reticular nucleus; integration of descending and ascending systems regulating voluntary forelimb movements. Front Comput Neurosci 9: 102, 2015.

Altman J. Postnatal development of the cerebellar cortex in the rat. 3. Maturation of the components of the granular layer. J Comp Neurol 145: 465-513, 1972a.

Altman J. Postnatal development of the cerebellar cortex in the rat. I. The external germinal layer and the transitional molecular layer. J Comp Neurol 145: 353-397, 1972b.

Altman J. Postnatal development of the cerebellar cortex in the rat. II. Phases in the maturation of Purkinje cells and of the molecular layer. J Comp Neurol 145: 399-463, 1972c.

Amarasingham A, Harrison MT, Hatsopoulos NG, and Geman S. Conditional modeling and the jitter method of spike resampling. J Neurophysiol 107: 517-531, 2012.

Andjus PR, Zhu L, Cesa R, Carulli D, and Strata P. A change in the pattern of activity affects the developmental regression of the Purkinje cell polyinnervation by climbing fibers in the rat cerebellum. Neuroscience 121: 563-572, 2003.

Arshavsky YI, Gelfand IM, Orlovsky GN, and Pavlova GA. Messages conveyed by spinocerebellar pathways during scratching in the cat. I. Activity of neurons of the lateral reticular nucleus. Brain Res 151: 479-491, 1978.

Azim E, and Alstermark B. Skilled forelimb movements and internal copy motor circuits. Curr Opin Neurobiol 33: 16-24, 2015.

Azim E, Jiang J, Alstermark B, and Jessell TM. Skilled reaching relies on a V2a propriospinal internal copy circuit. Nature 508: 357-363, 2014.

Benington JH, Woudenberg MC, and Heller HC. Apamin, a selective SK potassium channel blocker, suppresses REM sleep without a compensatory rebound. Brain Res 692: 86-92, 1995.

Blakemore SJ, Frith CD, and Wolpert DM. The cerebellum is involved in predicting the sensory consequences of action. Neuroreport 12: 1879-1884, 2001.

Blakemore SJ, Wolpert D, and Frith C. Why can't you tickle yourself? Neuroreport 11: R11-16, 2000.

Blumberg MS. Beyond dreams: do sleep-related movements contribute to brain development? Front Neurol 1: 140, 2010.

Blumberg MS, and Dooley JC. Phantom limbs, neuroprosthetics, and the developmental origins of embodiment. Trends Neurosci 40: 603-612, 2017.

Blumberg MS, Marques HG, and lida F. Twitching in sensorimotor development from sleeping rats to robots. Curr Biol 23: R532-537, 2013.

Blumberg MS, Sokoloff G, Tiriac A, and Del Rio-Bermudez C. A valuable and promising method for recording brain activity in behaving newborn rodents. Dev Psychobiol 57: 506-517, 2015. 
Brooks JX, Carriot J, and Cullen KE. Learning to expect the unexpected: rapid updating in primate cerebellum during voluntary self-motion. Nat Neurosci 18: 1310-1317, 2015.

Chung L. A brief introduction to the transduction of neural activity into Fos signal. Dev Reprod 19: 61-67, 2015.

Crapse TB, and Sommer MA. Corollary discharge across the animal kingdom. Nat Rev Neurosci 9: 587-600, 2008.

Cullen KE. Sensory signals during active versus passive movement. Curr Opin Neurobiol 14: 698-706, 2004.

Dale A, and Cullen KE. The ventral posterior lateral thalamus preferentially encodes externally applied versus active movement: Implications for self-motion perception. Cereb Cortex 1-14, 2017.

Davis WJ, Siegler MV, and Mpitoses. Distributed neuronal oscillators and efference copy in the feeding system of Pleurobranchaea. J Neurophysiol 36: 258-274, 1973.

de Zeeuw Cl, Holstege JC, Calkoen F, Ruigrok TJ, and Voogd J. A new combination of WGA-HRP anterograde tracing and GABA immunocytochemistry applied to afferents of the cat inferior olive at the ultrastructural level. Brain Res 447: 369-375, 1988.

De Zeeuw CI, Simpson JI, Hoogenraad CC, Galjart N, Koekkoek SK, and Ruigrok TJ. Microcircuitry and function of the inferior olive. Trends Neurosci 21: 391-400, 1998.

Del Rio-Bermudez C, Plumeau AM, Sattler NJ, Sokoloff G, and Blumberg MS. Spontaneous activity and functional connectivity in the developing cerebellorubral system. J Neurophysiol 116: 1316-1327, 2016.

Del Rio-Bermudez C, Sokoloff G, and Blumberg MS. Sensorimotor processing in the newborn rat red nucleus during active sleep. J Neurosci 35: 8322-8332, 2015.

Devor A. The great gate: control of sensory information flow to the cerebellum. Cerebellum 1: 27-34, 2002.

Diamond A. Close interrelation of motor development and cognitive development and of the cerebellum and prefrontal cortex. Child Dev 71: 44-56, 2000.

Evans CG, Jing J, Proekt A, Rosen SC, and Cropper EC. Frequency-dependent regulation of afferent transmission in the feeding circuitry of Aplysia. $J$ Neurophysiol 90: 3967-3977, 2003.

Fee MS, Mitra PP, and Kleinfeld D. Central versus peripheral determinants of patterned spike activity in rat vibrissa cortex during whisking. J Neurophysiol 78: 1144-1149, 1997.

Feinberg I, and Guazzelli M. Schizophrenia--a disorder of the corollary discharge systems that integrate the motor systems of thought with the sensory systems of consciousness. Br J Psychiatry 174: 196-204, 1999.

Feldman DE. The spike-timing dependence of plasticity. Neuron 75: 556-571, 2012.

Ford JM, Roach BJ, Faustman WO, and Mathalon DH. Out-of-synch and out-of-sorts: dysfunction of motor-sensory communication in schizophrenia. Biol Psychiatry 63: 736743, 2008.

Fukushima K. The interstitial nucleus of Cajal in the midbrain reticular formation and vertical eye movement. Neurosci Res 10: 159-187, 1991.

Garden DL, Rinaldi A, and Nolan MF. Active integration of glutamatergic input to the inferior olive generates bidirectional postsynaptic potentials. J Physiol 595: 1239-1251, 2017.

Gellman R, Gibson AR, and Houk JC. Inferior olivary neurons in the awake cat: detection of contact and passive body displacement. J Neurophysiol 54: 40-60, 1985. 
Gellman R, Houk JC, and Gibson AR. Somatosensory properties of the inferior olive of the cat. J Comp Neurol 215: 228-243, 1983.

Gramsbergen A, Schwartze P, and Prechtl HF. The postnatal development of behavioral states in the rat. Dev Psychobiol 3: 267-280, 1970.

Gui L, LaGrange LP, Larson RA, Gu M, Zhu J, and Chen QH. Role of small conductance calcium-activated potassium channels expressed in PVN in regulating sympathetic nerve activity and arterial blood pressure in rats. Am J Physiol Regul Integr Comp Physiol 303: R301-310, 2012.

Gymnopoulos M, Cingolani LA, Pedarzani P, and Stocker M. Developmental mapping of small-conductance calcium-activated potassium channel expression in the rat nervous system. J Comp Neurol 522: 1072-1101, 2014.

Harrison MT, and Geman S. A rate and history-preserving resampling algorithm for neural spike trains. Neural Comput 21: 1244-1258, 2009.

Horn KM, Pong M, and Gibson AR. Discharge of inferior olive cells during reaching errors and perturbations. Brain Res 996: 148-158, 2004.

Huang CC, Sugino K, Shima Y, Guo C, Bai S, Mensh BD, Nelson SB, and Hantman AW. Convergence of pontine and proprioceptive streams onto multimodal cerebellar granule cells. Elife 2: e00400, 2013.

Jouvet-Mounier D, Astic L, and Lacote D. Ontogenesis of the states of sleep in rat, cat, and guinea pig during the first postnatal month. Dev Psychobiol 2: 216-239, 1970.

Kakizawa S, Yamasaki M, Watanabe M, and Kano M. Critical period for activitydependent synapse elimination in developing cerebellum. J Neurosci 20: 4954-4961, 2000.

Kano M, and Hashimoto K. Activity-dependent maturation of climbing fiber to Purkinje cell synapses during postnatal cerebellar development. Cerebellum 11: 449-450, 2012.

Karlsson KA, Gall AJ, Mohns EJ, Seelke AM, and Blumberg MS. The neural substrates of infant sleep in rats. PLoS Biol 3: e143, 2005.

Kawamura Y, Nakayama H, Hashimoto K, Sakimura K, Kitamura K, and Kano M. Spike timing-dependent selective strengthening of single climbing fibre inputs to Purkinje cells during cerebellar development. Nat Commun 4: 2732, 2013.

Keating JG, and Thach WT. Nonclock behavior of inferior olive neurons: interspike interval of Purkinje cell complex spike discharge in the awake behaving monkey is random. J Neurophysiol 73: 1329-1340, 1995.

Kirk MD, and Wine JJ. Identified interneurons produce both primary afferent depolarization and presynaptic inhibition. Science 225: 854-856, 1984.

Kreider JC, and Blumberg MS. Mesopontine contribution to the expression of active 'twitch' sleep in decerebrate week-old rats. Brain Res 872: 149-159, 2000.

Kremkow J, Perrinet LU, Masson GS, and Aertsen A. Functional consequences of correlated excitatory and inhibitory conductances in cortical networks. J Comput Neurosci 28: 579-594, 2010.

Lakke EA. The projections to the spinal cord of the rat during development: a timetable of descent. Adv Anat Embryol Cell Biol 135: I-XIV, 1-143, 1997.

Lang EJ, Apps R, Bengtsson F, Cerminara NL, De Zeeuw Cl, Ebner TJ, Heck DH, Jaeger D, Jorntell H, Kawato M, Otis TS, Ozyildirim O, Popa LS, Reeves AM, Schweighofer N, Sugihara I, and Xiao J. The roles of the olivocerebellar pathway in motor learning and motor control. A consensus paper. Cerebellum 16: 230-252, 2017. 
Lang EJ, Sugihara I, and Llinas R. Differential roles of apamin- and charybdotoxinsensitive $\mathrm{K}+$ conductances in the generation of inferior olive rhythmicity in vivo. $J$ Neurosci 17: 2825-2838, 1997.

Lee HS, and Mihailoff GA. Convergence of cortical and cerebellar projections on single basilar pontine neurons: a light and electron microscopic study in the rat. Neuroscience 39: 561-577, 1990.

Li WC, Higashijima S, Parry DM, Roberts A, and Soffe SR. Primitive roles for inhibitory interneurons in developing frog spinal cord. J Neurosci 24: 5840-5848, 2004.

Mason P. Medical Neurobiology. New York: Oxford University Press, 2011.

McVea DA, Murphy TH, and Mohajerani MH. Large scale cortical functional networks associated with slow-wave and spindle-burst-related spontaneous activity. Front Neural Circuits 10: 103, 2016.

Morris R, Vallester KK, Newton SS, Kearsley AP, and Whishaw IQ. The differential contributions of the parvocellular and the magnocellular subdivisions of the red nucleus to skilled reaching in the rat. Neuroscience 295: 48-57, 2015.

Mukherjee D, Yonk AJ, Sokoloff G, and Blumberg MS. Wakefulness suppresses retinal wave-related neural activity in visual cortex. J Neurophysiol 118: 1190-1197, 2017.

Nelson BJ, and Mugnaini E. The rat inferior olive as seen with immunostaining for glutamate decarboxylase. Anat Embryol (Berl) 179: 109-127, 1988.

Nicholson DA, and Freeman JH, Jr. Addition of inhibition in the olivocerebellar system and the ontogeny of a motor memory. Nat Neurosci 6: 532-537, 2003.

Onodera S, and Hicks TP. A comparative neuroanatomical study of the red nucleus of the cat, macaque and human. PLoS One 4: e6623, 2009.

Onodera S, and Hicks TP. A projection linking motor cortex with the LM-suprageniculate nuclear complex through the periaqueductal gray area which surrounds the nucleus of Darkschewitsch in the cat. Prog Brain Res 112: 85-98, 1996.

Pivetta C, Esposito MS, Sigrist M, and Arber S. Motor-circuit communication matrix from spinal cord to brainstem neurons revealed by developmental origin. Cell 156: 537-548, 2014.

Poulet JF, and Hedwig B. The cellular basis of a corollary discharge. Science 311: 518522, 2006.

Poulet JF, and Hedwig B. New insights into corollary discharges mediated by identified neural pathways. Trends Neurosci 30: 14-21, 2007.

Puro DG, and Woodward DJ. Maturation of evoked climbing fiber input to rat cerebellar purkinje cells (I.). Exp Brain Res 28: 85-100, 1977a.

Puro DG, and Woodward DJ. Maturation of evoked mossy fiber input to rat cerebellar Purkinje cells (II.). Exp Brain Res 28: 427-441, $1977 \mathrm{~b}$.

Requarth T, and Sawtell NB. Plastic corollary discharge predicts sensory consequences of movements in a cerebellum-like circuit. Neuron 82: 896-907, 2014.

Roffwarg HP, Muzio JN, and Dement WC. Ontogenetic development of the human sleep-dream cycle. Science 152: 604-619, 1966.

Ruigrok TJ, Sillitoe RV, and Voogd J. Cerebellum and cerebellar connections In: The Rat Nervous System, edited by Paxinos G. UK: Elsevier Academic Press, 2014, p. 133204.

Saint-Cyr JA. The projection from the motor cortex to the inferior olive in the cat. An experimental study using axonal transport techniques. Neuroscience 10: 667-684, 1983. 
Saint-Cyr JA, and Courville J. Sources of descending afferents to the inferior olive from the upper brain stem in the cat as revealed by the retrograde transport of horseradish peroxidase. J Comp Neurol 198: 567-581, 1981.

Schneider DM, Nelson A, and Mooney R. A synaptic and circuit basis for corollary discharge in the auditory cortex. Nature 513: 189-194, 2014.

Sedgwick EM, and Williams TD. Responses of single units in the inferior olive to stimulation of the limb nerves, peripheral skin receptors, cerebellum, caudate nucleus and motor cortex. J Physiol 189: 261-279, 1967.

Seelke AMH, and Blumberg MS. The form and function of infant sleep: From muscle to neocortex. In: The Oxford Handbook of Developmental Behavioral Neuroscience, edited by Blumberg MS, Freeman JH, and Robinson SR. New York: Oxford University Press, 2010, p. 391-423.

Sgritta M, Locatelli F, Soda T, Prestori F, and D'Angelo EU. Hebbian Spike-Timing Dependent Plasticity at the Cerebellar Input Stage. J Neurosci 37: 2809-2823, 2017.

Shimono T, Nosaka S, and Sasaki K. Electrophysiological study on the postnatal development of neuronal mechanisms in the rat cerebellar cortex. Brain Res 108: 279294, 1976.

Sokoloff G, Plumeau AM, Mukherjee D, and Blumberg MS. Twitch-related and rhythmic activation of the developing cerebellar cortex. J Neurophysiol 114: 1746-1756, 2015a.

Sokoloff G, Uitermarkt BD, and Blumberg MS. REM sleep twitches rouse nascent cerebellar circuits: Implications for sensorimotor development. Dev Neurobiol 75: 11401153, $2015 b$.

Sommer MA, and Wurtz RH. Brain circuits for the internal monitoring of movements. Annu Rev Neurosci 31: 317-338, 2008.

Sommer MA, and Wurtz RH. A pathway in primate brain for internal monitoring of movements. Science 296: 1480-1482, 2002.

Sommer MA, and Wurtz RH. What the brain stem tells the frontal cortex. I. Oculomotor signals sent from superior colliculus to frontal eye field via mediodorsal thalamus. $J$ Neurophysiol 91: 1381-1402, 2004.

Tiriac A, and Blumberg MS. Gating of reafference in the external cuneate nucleus during self-generated movements in wake but not sleep. Elife 5: 2016.

Tiriac A, Del Rio-Bermudez C, and Blumberg MS. Self-generated movements with "unexpected" sensory consequences. Curr Biol 24: 2136-2141, 2014.

Tiriac A, Sokoloff G, and Blumberg MS. Myoclonic Twitching and Sleep-Dependent Plasticity in the Developing Sensorimotor System. Curr Sleep Med Rep 1: 74-79, 2015.

Tiriac A, Uitermarkt BD, Fanning AS, Sokoloff G, and Blumberg MS. Rapid whisker movements in sleeping newborn rats. Curr Biol 22: 2075-2080, 2012.

van Kan PL, Gibson AR, and Houk JC. Movement-related inputs to intermediate cerebellum of the monkey. J Neurophysiol 69: 74-94, 1993.

Wang SS, Kloth AD, and Badura A. The cerebellum, sensitive periods, and autism. Neuron 83: 518-532, 2014.

Wang VY, and Zoghbi HY. Genetic regulation of cerebellar development. Nat ReV Neurosci 2: 484-491, 2001.

Watanabe M, and Kano M. Climbing fiber synapse elimination in cerebellar Purkinje cells. Eur J Neurosci 34: 1697-1710, 2011. 
Welsh JP, Lang EJ, Suglhara I, and Llinas R. Dynamic organization of motor control within the olivocerebellar system. Nature 374: 453-457, 1995.

Williams PT, Kim S, and Martin JH. Postnatal maturation of the red nucleus motor map depends on rubrospinal connections with forelimb motor pools. J Neurosci 34: 4432-4441, 2014.

Wolpert DM, Miall RC, and Kawato M. Internal models in the cerebellum. Trends Cogn Sci 2: 338-347, 1998.

Xu W, Jones S, and Edgley SA. Event time representation in cerebellar mossy fibres arising from the lateral reticular nucleus. J Physiol 591: 1045-1062, 2013.

Yang JW, An S, Sun JJ, Reyes-Puerta V, Kindler J, Berger T, Kilb W, and Luhmann HJ. Thalamic network oscillations synchronize ontogenetic columns in the newborn rat barrel cortex. Cereb Cortex 23: 1299-1316, 2013.

Yang Y, Cao P, Yang Y, and Wang SR. Corollary discharge circuits for saccadic modulation of the pigeon visual system. Nat Neurosci 11: 595-602, 2008.

Zuk A, Rutherford JG, and Gwyn DG. Projections from the interstitial nucleus of Cajal to the inferior olive and to the spinal cord in cat: a retrograde fluorescent double-labeling study. Neurosci Lett 38: 95-101, 1983. 